\title{
Deformation of aluminum in situ SEM and full field measurements by digital image correlation: evidence of concomitant crystal slip and grain boundary sliding

\author{
A. Dimanova, A. El Sabbagh ${ }^{\mathrm{a}}$, J. Raphanel ${ }^{\mathrm{a}}$, T.N. Lê, M. Bornert ${ }^{\mathrm{b}}$, S. Hallais ${ }^{\mathrm{a}}$, A. Tanguy \\ ${ }^{b}$ Laboratoire Navier, CNRS UMR 8205, Ecole des Ponts Paristech, 77420 Champs sur Marne, France
} \\ ${ }^{a}$ Laboratoire de Mécanique des Solides, CNRS UMR 7649, École Polytechnique, 91128, Palaiseau Cedex, France.
}

\begin{abstract}
Mechanical testing in situ scanning electron microscopy (SEM) has become a current technique for multiscale micromechanical investigation of polycrystalline materials, because direct observation of deformation microstructures allows identification of strain heterogeneities and related mechanisms. Yet, most of the studies are based on the inherited post mortem microstructures, thus precluding to unravel the local loading history, to understand the development of localization patterns, the potential interactions of concomitant mechanisms and to quantify their respective contributions to the overall strain. We therefore developed a novel experimental setup for thermomechanical testing in situ SEM, especially suited for full strain field measurements based on digital image correlation (DIC) from the sample scale, to the scales of the aggregate and the single grain. We present results obtained during simple compression, at controlled displacement rate and at temperatures up to $400^{\circ} \mathrm{C}$, for polycrystalline aluminum presenting randomly oriented coarse grains (ca. $300 \mu \mathrm{m}$ in size). According to the different scales of interest, specific surface marking patterns were realized by electron microlithography. Kinematic analysis by digital image correlation (DIC) allowed to retrieve full surface strain fields. The latter evidenced that the overall viscoplastic response was dominated by crystal slip plasticity. Increasing temperature favored the activation of non-octahedral slip, but also substantial and continuous contribution of grain boundary sliding (GBS). We suggest the latter mechanism as necessary to accommodate local plastic incompatibilities between neighboring grains.
\end{abstract}




\section{Graphical abstract:}
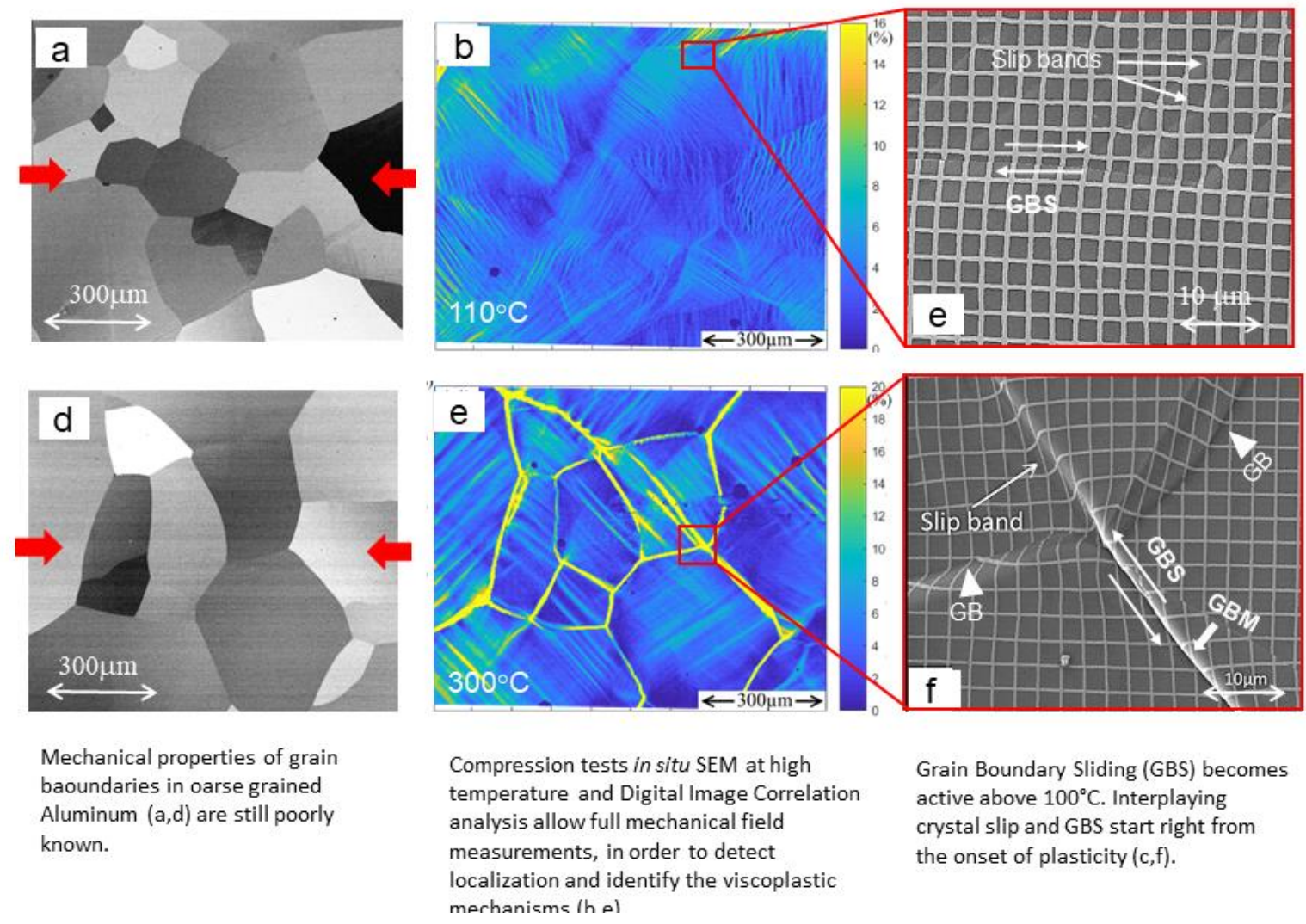

Mechanical properties of grain baoundaries in oarse grained Aluminum (a,d) are still poorly known.
Grain Boundary Sliding (GBS) becomes active above $100^{\circ} \mathrm{C}$. Interplaying crystal slip and GBS start right from the onset of plasticity $(c, f)$.

Key words: Aluminum, in situ SEM testing, Digital Image Correlation, Crystal plasticity, Grain boundary sliding. 


\section{Introduction}

Understanding and modeling viscoplastic deformation of polycrystalline materials is essential for the control of shape forming processes and more generally for estimation of the durability of functional metallic structures submitted to extreme thermomechanical solicitations. Viscoplasticity of polycrystalline metals and alloys involves concomitantly several physical mechanisms operating in volume, such as twinning and crystal slip, or at interfaces and grain boundaries, such as grain sliding and/or migration. At high temperatures crystal slip is accompanied by recovery processes such as dislocation climb, polygonization and recrystallization, which are controlled by diffusional mass transfer. In cases of superplastic behavior, especially for fine grain sizes and low strain rates, the latter may account as a dominant mechanism per se. The respective contributions of the different mechanisms to the overall strain depend on the considered thermodynamic and loading conditions (e.g. temperature, stress and/or strain rate magnitudes, chemical environment), but also microstructure (grain size and morphology, texture, second phases). For a given material and conditions, one may determine the dominant mechanisms on the basis of the widely used concept of deformation mechanisms maps, introduced in the seventies [1], [2], [3]. However, the construction of such diagrams implicitly assumes that the considered mechanisms are independent, that is acting in parallel and contributing additively to the overall strain. This assumption is not straightforward, because simultaneously occurring mechanisms necessarily interact and some may possibly cooperate. In the case of dislocation creep ([4], [5]) diffusion controlled dislocation climb allows for recovery, and thus conditions the crystal glide efficiency and the overall steady-state creep rate. For instance, decades ago was already observed substantial activation of grain boundary sliding even for coarse grained polycrystals, which are predestined accordingly to deformation mechanisms maps solely to crystal slip ([6], [7]). In fact, macro-homogeneous flow can hardly be achieved exclusively based on crystal slip, because of the inherent anisotropy of crystal plasticity. Grain boundaries may act as efficient barriers to slip transfer from a grain to another, thus inducing local plastic incompatibilities ([8], [9], [10], [11]). However, the latter may be strongly reduced by modest contributions of grain boundary related mechanisms, such as grain boundary sliding [12], [13] and/or migration, involving diffusional mass transfer or mobile disconnections [14]. Realistic modeling of polycrystalline viscoplasticity is therefore subordinated to detailed understanding of the interactions between the different active mechanisms. However, even when different mechanisms are identified through their specific microstructural signatures, their chronology of activation and interactions remain unclear, if the microstructural observations are realized post mortem. Instead, it is necessary to identify and quantify these processes by in situ monitoring at the appropriate scales. Full mechanical field measurement based on DIC is the most suited experimental technique for such in situ micromechanical characterization. It allows one to access to the global mean mechanical response, but 
also to evidence multi-scale localization phenomena from the sample scale to the scale of the individual crystalline grains, that is from the structure to the intimate microstructure. For instance, in the latter case detection of localization allows one to identify the active mechanisms, to characterize their interactions and to calculate their respective contributions ([12], [13], [15]).

Over the last decade we developed different setups for in situ thermomechanical testing in a SEM, specifically devoted to full mechanical field measurements ([12], [13]). The devices allow to perform simple compression/traction and bending tests at both room and high temperatures. The principle of full field measurements by DIC relies on the presence of local contrasts within the material. Each material point may be defined uniquely based on a specific signature in terms of the surrounding local gray scale levels. These signatures are used as kinematic markers in order to determine the displacement fields during the mechanical transformation. The contrasts can be natural [15] or artificial [16]. For single phase polycrystalline materials observed in SEM there are not enough natural contrasts. We have therefore developed different techniques to provide surface kinematic markers, according to the microstructure and the testing conditions. The most recent technique consists in dewetting by annealing previously deposited thin metallic films in order to obtain randomly dispersed metallic spheroids onto the observed surface ([12], [13], [17]). Depending on metal, film thickness, temperature and atmosphere the size and the density of spheroids can be controlled, which makes it particularly suitable for very fine grained microstructures [17]. In the present work we studied nearly pure aluminum, containing only $0.1 \mathrm{wt} \% \mathrm{Mn}$ (kindly provided by Romain Quey). The starting material was extracted from the central part of laminated slabs. Heat treatment at $500^{\circ} \mathrm{C}$ resulted in an equilibrated and untextured microstructure, with coarse grain sizes reaching several hundreds of micrometers. We therefore applied the classical electron microlithography in order to produce regular golden micro-grids onto the sample surface [16].

For the mechanical testing, we used an original setup that we specifically developed for in situ SEM simple compression at temperatures of up to $600^{\circ} \mathrm{C}$. The apparatus design allows for observation over the entire specimen surface. Contact-less thermal monitoring and control is ensured by in situ infrared pyrometry. Parallelepiped samples $4 \times 4 \times 6 \mathrm{~mm}^{3}$ were axially shortened stepwise up to $10 \%$ at temperatures ranging between room conditions and $400^{\circ} \mathrm{C}$. High quality SEM images with several magnifications were acquired at each deformation step and further analyzed by DIC (CorelManuV software), in order to obtain kinematic surface fields and calculate full in plane strain fields ([18], [19], [20], [12]). The experimental details and analytical procedures are given in the following sections.

\section{Experimental and analytical procedures}




\subsection{In-situ SEM thermomechanical testing stage}

The mechanical loading part of the experimental device is based on a previously used homemade tractioncompression rig ([17], [15], [16], [13], [12]), which fits to the original sample holder stage of the SEM. The latter is a QUANTA 650 FEG-ESEM, equipped for EBSD (electron back scatter diffraction) analysis (HKL Nordlys II S camera is operated with Aztek 3.1 and data are post-processed with Channel 5 software). The rig frame is machined out of stainless steel and is U-shaped in order to provide maximum accessibility to the loading column and the sample (Fig. 1 and 2). Compression is driven by a conventional DC-motor Mattke 3557-K024C (nominal rotation speed and torque of $3000 \mathrm{rpm}$ and 0.040 N.m, respectively). A gearbox reduces the rotation speed while increasing the torque. Rotation transforms into translation thanks to a screw nut system and the crosshead glides on linear ball bearing along two stainless steel shafts. The crosshead is instrumented with a linear displacement sensor (optical rule type from Solartron), which is fixed on the frame and measures the displacements for a homemade controller allowing to apply loading rates as low as $0.1 \mu \mathrm{ms}^{-1}$. The loading capacity is $5 \mathrm{kN}$. The applied force is measured by a load cell from Ametek, inserted within the assembly of the loading column (Fig. 2). The compression stroke ranges within $12 \mathrm{~mm}$.

The main technical developments in the present work are concerned with the heating parts and temperature monitoring. Our previous heating setup was based on radiative heat transfer, thanks to a tubular furnace enclosing sample and pistons, but only a restricted sample area was accessible to observation through a circular window [12]. In contrast, the present system is designed to offer the entire sample surface free for observation. For that purpose, two tubular furnaces are fitted to the pistons and the sample is solely heated by conduction. The two assemblies of pistons and their tubular resistors are thermally insulated by cylindrical ceramic shields (machined from pyrophylite). We used $\mathrm{SiC}$ pistons which are connected to the rig frame with metallic wires, hence avoiding electrical charging of samples. Precise temperature monitoring is usually obtained by attaching or directly welding a thermocouple on the sample. In order to avoid any risk of local alloying, or loss of contact during sample deformation we did not apply such technique. Temperature monitoring and control is ensured by contactless measurements realized with an infrared pyrometer installed within the SEM chamber and targeting the sample from aside (Fig. 1). The tests last several hours, so that a cooling circuit (mix of water and alcohol) is established to protect the electronics, load cell and actuator. The cooling circuit is composed of flexible silicone tubes connecting two stainless steel parts and a chiller centrifugal pump providing continuous cooling flow, the temperature of which is adjusted according to the experimental set point. For example, when the sample is heated at $300{ }^{\circ} \mathrm{C}$ we regulate the water temperature at $10{ }^{\circ} \mathrm{C}$. One of the water cooled steel elements is inserted in-between the insulated heating element and the load-cell, which is attached to the frame. The other one is placed in-between the second insulated heating element and the cross-head of the loading column. 
All the elements of the latter are precisely aligned in order to avoid stress concentrations at the ceramic parts. The temperature of the load-cell is monitored by a classical RTD Pt100 sensor.
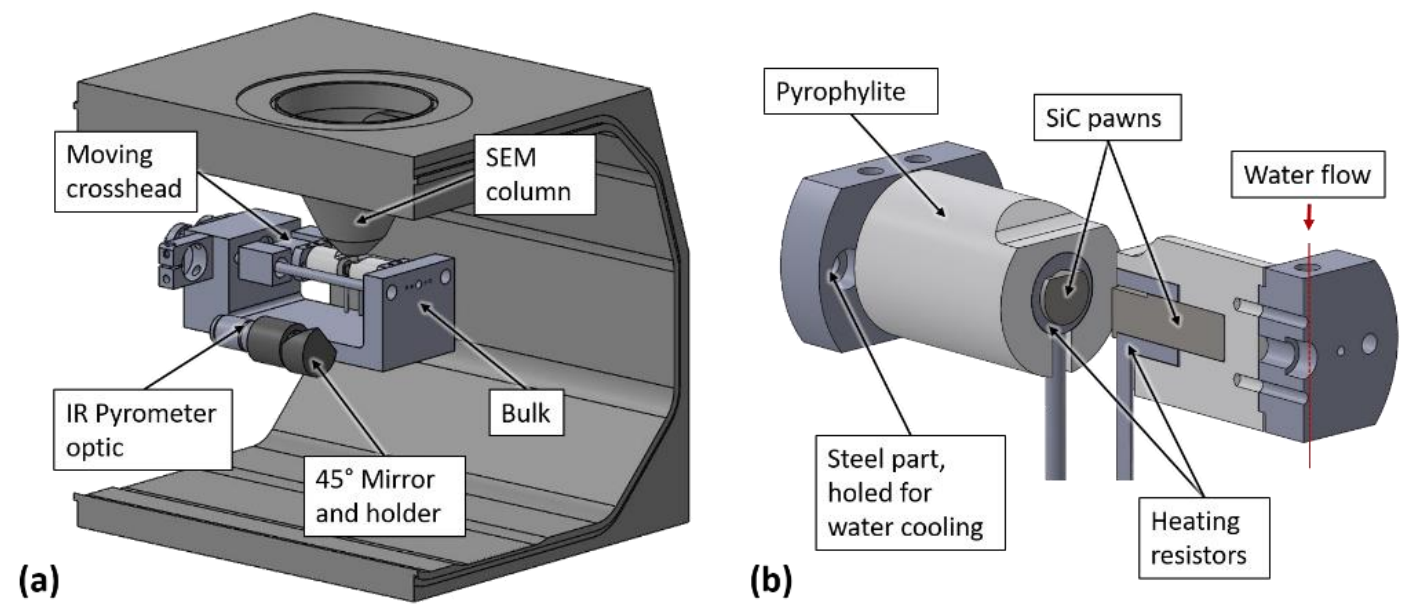

Figure 1: (a) Schematic view of the SEM chamber with the in-situ testing set up, composed of the deformation machine and the IR-pyrometer. (b) Magnification of the deformation column assembly, showing the two steel cross-heads with two SiC pistons surrounded by two heating coils (Pt resistor wires) and annealed pyrophilite thermal shielding. The cross heads comprise a liquid cooling circuit.

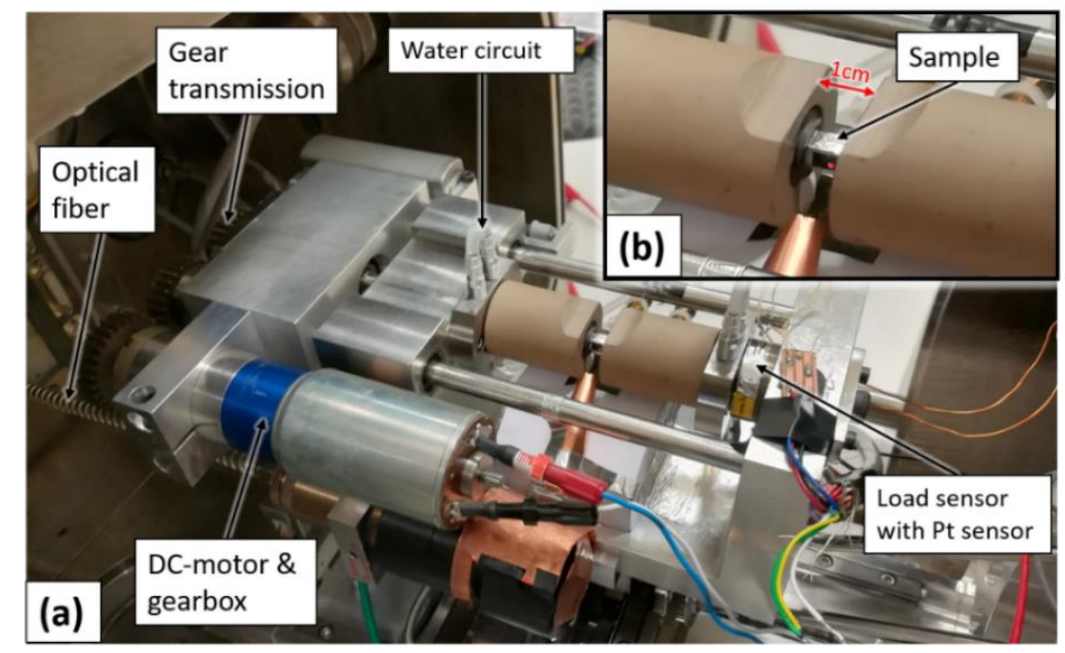

(c)

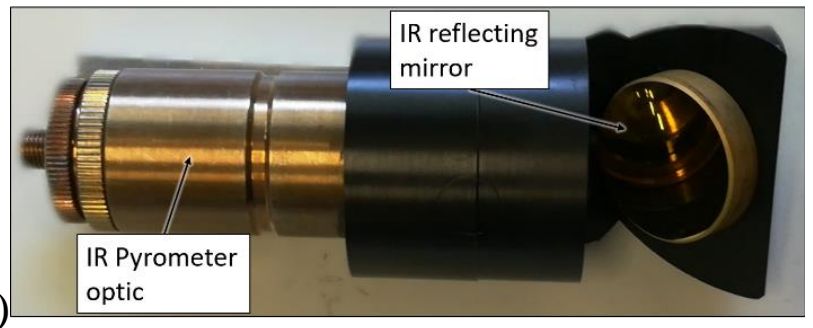

Figure 2: (a) Overal view of the SEM chamber with the in-situ testing machine mounted on the SEM platen. The pyrometer lies aside, under the mator. The steel elements of the fixed and mobile crosshead comprise cooling fluid circuits (b) Zoom on the central part of the loading column, composed of pyrophylite insulators, shielding the Pt resistor coils, which surround the SiC pistons. c: Optics and Lumasens IGA 320/23-LO pyrometer. The IR reflecting mirror is mounted on the black MACOR tilted holder. The whole assembly mounts on the mechanical testing stage, under the motor, as shown in (a). In order to avoid parasite IR radiations, the holder of the reflecting mirror is partially covered with copper foil. The mirror collects exclusively the radiations emitted by the targeted sample side, which is painted in black. In (b) one can see the red spot on the sample side where the pyrometer is focused. 
A schematic diagram of the heating elements is shown on Fig. 1(b). The heat is generated by two heating resistors with double sense wiring of $1.1 \Omega$ each $(40 \mathrm{~W})$. The double sense wiring precludes development of magnetic fields which could disturb the electron beam. The resistors consist of platinum wires $(0.3 \mathrm{~mm}$ in diameter) embedded in multi-layered ceramic insulation. The resulting tubular heating elements are $14 \mathrm{~mm}$ in length, with 7 and $13 \mathrm{~mm}$ inner and outer diameters, respectively. PID controlled current is provided by a switch-mode DC power supply (maximum output: 20V/20A). The heating elements are placed surrounding two sintered $\mathrm{SiC}$ pistons, chosen for their appropriate mechanical strength and thermal conductivity. Symmetrical heating of the sample is obtained by conduction through the pistons as shown in Figures $1 \mathrm{~b}$ and $2 \mathrm{~b}$. The latter have precisely flat and mirror polished end surfaces to ensure good contact and heat transfer to the sample, but also to reduce interfacial frictions. Insulating shields made of annealed pyrophyllite (aluminosilicate ceramic) limit radiative loss. The starting pyrophyllite material is soft and easy to machine in its naturally hydrated form. After machining the shield pieces are dehydrated by annealing at $1300{ }^{\circ} \mathrm{C}$ for about 4 hours, reaching a compressive strength of the order of $800 \mathrm{MPa}$. The complete experimental setup is shown in Figures 1 and 2. The details on the in situ SEM pyrometer are given in the following section.

\subsection{Contactless temperature measurement and monitoring}

The measurement of sample temperature without contact is a key development of our setup. On the one hand, the geometrical constraints of the heating concept preclude introducing a thermocouple close enough to the sample and leaving accessible the whole observation area. On the other hand, the aluminum sample and the experimental temperature range do not allow for easy welding or sticking a thermocouple on the sample back side without risk of losing contact during loading. Therefore, we adapted an infrared pyrometer Lumasens IGA 320/23-LO for in situ SEM contactless measurements of sample temperature. The infrared radiation emitted by the latter is transmitted by a mirror to the optics of the pyrometer located in the SEM chamber, which is connected via an optical fiber to the detector and electronics located outside the SEM (Fig. 2). The optics' focal distance can vary from $8.8 \mathrm{~cm}$ to $11 \mathrm{~cm}$, which allows an accurate focus on the sample's surface with a spot size of $0.8 \mathrm{~mm}$. The considered wave lengths range between 2 and $2.6 \mathrm{~mm}$. Temperature measurement ranges between $100^{\circ} \mathrm{C}$ and $700^{\circ} \mathrm{C}$, with an overall estimated precision within

$3{ }^{\circ} \mathrm{C}$. A mirror deflects the infrared radiations from the sample to the pyrometer optics, which are clamped aside the rig (Fig. 2). The pyrometer optics and the deflecting mirror are shown on Figure 3. The optical axis is aligned at $60^{\circ}$ to the normal of the sample's side. The deflecting mirror is protected by a copper shield, in order to avoid parasite emissions from the chamber, the setup and more specifically from the heating elements. The only measured infrared signal is collected by a copper funnel operating as a waveguide from the sample side to the deflecting mirror (Fig. 2). 
According to the Stefan-Boltzmann law the irradiance is proportional to the fourth power of the temperature. Therefore, the reading of the pyrometer corresponds to the intensity of infrared radiations within the considered wavelength range emitted by the sample. The emissivity coefficient $\varepsilon$ of the sample represents the ratio of its real radiative emission and that of the theoretical black body. Together with the Stefan-Boltzmann constant it represents the proportionality between radiation intensity and temperature. The higher is $\varepsilon$ and the better is the measurement. Metals are called selective bodies, because their emissivity coefficients are usually low, but also temperature and wavelength dependent. In the present case $\varepsilon$ may be assumed constant over the limited wavelength range. But for aluminum $\mathrm{e}=0,3$, which is very low. We improved the emissivity coefficient up to $\varepsilon=0,64$ by spraying thermoresistant black paint onto the sample surface targeted by the pyrometer optical line.

The pyrometer is initially calibrated for atmospheric conditions, that is at $10^{5} \mathrm{~Pa}$ total air pressure, that is in the presence of water vapor, which is very absorbing in the wavelength rage of interest. Therefore, we specifically calibrated the measurements for the high vacuum of the SEM chamber $\left(10^{-3} \mathrm{~Pa}\right)$. We actually established corrections to the emissivity coefficient for each experimental temperature. For that purpose, we prepared a replica aluminum sample, of dimensions identical to these of the samples used for mechanical testing. K-type thermocouple was inserted within a borehole of $0.5 \mathrm{~mm}$, that was drilled in the center of the sample. The corresponding temperature was used as reference for the pyrometer readout, while adjusting the emissivity coefficient parameter.

\subsection{Material and sample preparation}

The starting aluminum material is very pure, with only $0.1 \mathrm{wt} . \%$. of manganese. This alloying element reduces the mobility of grain boundaries by solute drag effect ([21], [22]). Thermal stability of the microstructure is important for in situ strain measurements for at least two reasons. First, migrating grain boundaries may perturb the surface marking, and hence interfere with the digital correlation process (described in the following), but also obscure the contribution of boundary sliding mechanism. Secondly, the correlation procedure compares an actual state with a reference one, and hence migrating boundaries and evolving microstructure would preclude cumulative strain analysis. The slightly alloyed material is a single phase polycrystal with a face centered cubic structure. These characteristics make possible a precise analysis of the micro-mechanisms that take place in the course of plastic deformation ([7], [8]). The material has low melting point $\left(667^{\circ} \mathrm{C}\right)$ and yield stress (a few MPa), and hence the setup allows to cover a wide range of experimental conditions and to activate different potential mechanisms ([6], [7], [8], [9], [10], [23]).

The starting material is received as cold rolled slabs. The samples are cut out from their central part in order to limit microstructural gradients due to boundary effects. Sample are parallelepiped shaped $\left(4 \times 4 \times 6 \mathrm{~mm}^{3}\right)$, with the 
rolling direction parallel to their longest dimension, which is also meant as the compression axis. Annealing at $500^{\circ} \mathrm{C}$ for 20 - 30 minutes strongly reduces the rolling texture, and results in a fairly homogeneous and equant recrystallized microstructure. In spite of the Mn solute, at such high temperature mobility of grain boundaries is fast enough, so that we obtain a coarse grained and equant microstructure, with mean grain size of $300 \mu \mathrm{m}$ (measured by intercept method) and a mean aspect ratio of 1.2. However, although unimodal, the grain size distribution remains large and grain diameters typically range between $100 \mu \mathrm{m}$ and $1000 \mu \mathrm{m}$.
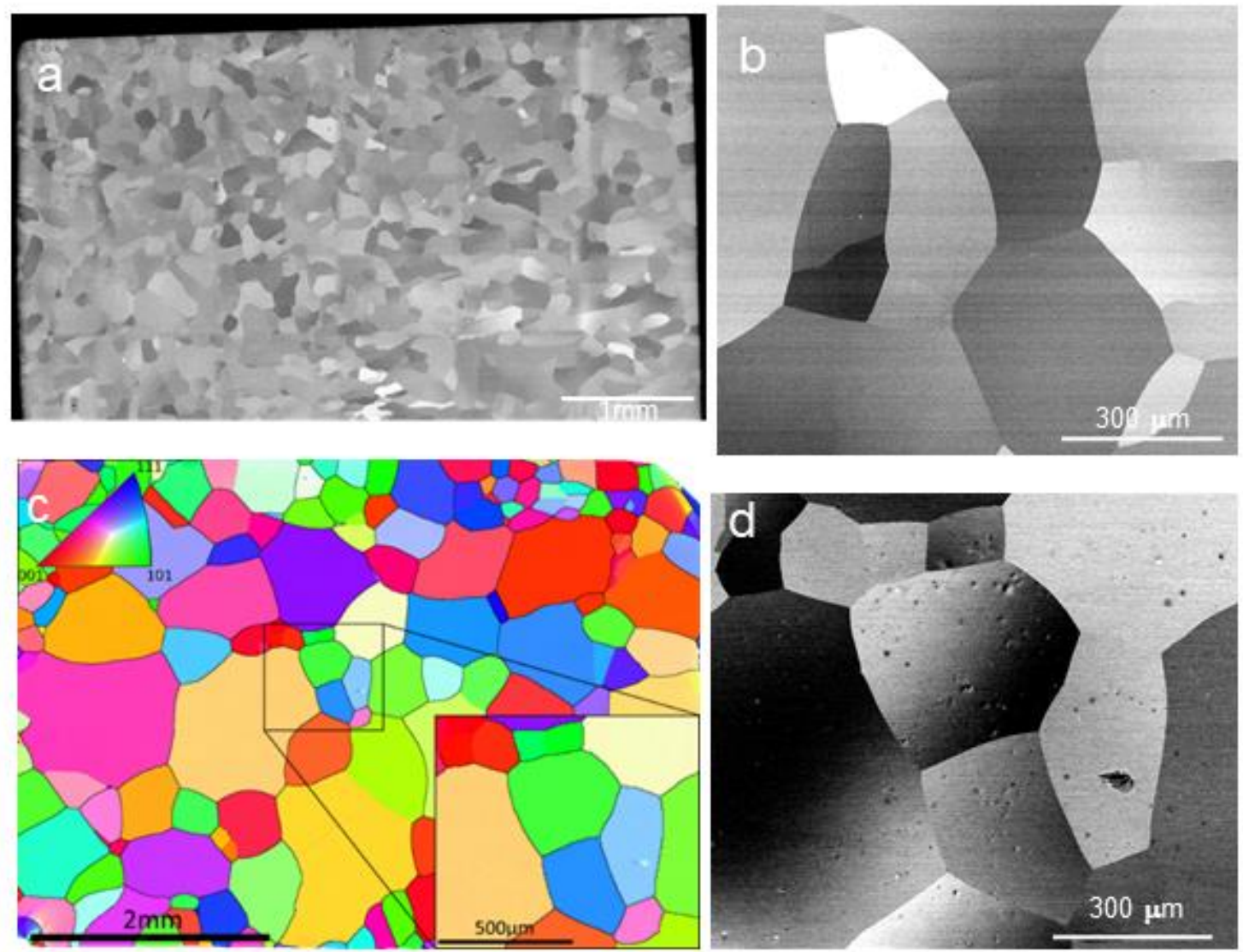

Figure 4: Sample microstructure. a: Large view of a typical aluminum sample (4x4x6 mm3), reconstructed from SEM micrographs in Z-contrast mode. b: Closer view of the microstructure (SEM Z-contrast mode). c: Inverse pole figure (IPF) map with respect to the compression axis (horizontal) from an EBSD scan of a sample surface $(4 \times 6 \mathrm{~mm} 2)$. The IPF color reference is inserted in the upper left corner. The central area of $1 \mathrm{~mm}^{2}$, enlarged at the lower right corner, is the main region of interest (ROI-1) which is studied in details by DIC and which SEM micrograph in Z-contrast mode is shown in (d). One can see some etch pits resulting from the electrolytic treatment which may sometimes perturb the EBSD indexations (see the North-Souh elongated light blue grain).

The annealed samples are first mechanically polished with $\mathrm{SiC}$ grinding papers down to 4000 grade ( $5 \mu \mathrm{m}$ particle) and secondly with diamond paste down to $3 \mu \mathrm{m}$. Mirror quality finish is obtained by electro-polishing with a commercial STRUERS A2 electrolyte solution. The procedure removes the cold worked zone, ensuring a good crystalline surface state adequate for EBSD (electron backscatter diffraction) analysis (Fig. 4a), but may result in some etch pits as shown in Figure 4d. For each sample we choose a representative region of interest (ROI), of $1 \mathrm{~mm}^{2}$ with homogeneous and non-textured microstructure, such in Figure $4 \mathrm{~b}$. 


\subsection{Digital image correlation}

The DIC (digital image correlation) technique consists in the comparison between an image representing an actual deformed state to an image representing a previous reference state. The procedure aims at the characterization of the mechanical transformation leading from the reference state to the actual one. In our case we applied 2D DIC, which implies solely the characterization of in plane displacements and corresponding deformation.

The present DIC method relies on the existence of contrasts, that is variable local grey scale levels, which can be assigned to the surficial material points as characteristic signatures. In practice the procedure aims to determine the surficial displacement field of material points that are discretely distributed following a periodic pattern like a grid. These measurement points are centered within restricted domains, called correlation windows, which grey scale level is considered as the discriminative signature of the measurement point. The in plane displacement field of the latter, corresponding to the mechanical transformation between the reference and the actual states, is obtained by the minimization of the so called correlation coefficient, defined on the basis of the grey scale levels of the reference and actual states. The corresponding in plane transformation gradient is computed following different local integration schemes, based on a contour integral defined by a chosen number (usually from 2 to 8 ) of nearest neighboring measurement points. The computation of the in plane strain components depends on strong hypothesis, like volume conservation, or axisymmetric deformation. The accuracy of strain computation increases with the number of considered neighboring points (large contour integral). Conversely, the resolution of the computed strain field decreases due to the increasing local gage length (for details see [12]).

In our case, the local contrasts are provided artificially. We applied SEM micro-lithography for the deposition of golden grid patterns onto the observed surfaces of the samples. The technique consists of several steps. At first, a polymer layer is deposited on the sample surface, which constant thickness is achieved by centrifugation. The polymer is then irradiated with the electron beam of the SEM following a specific pattern. The latter is usually a simple regular grid ([16]). In our case it could be any type of regular or random pattern, because we use a Raith system allowing precise control of the SEM electron beam. Secondly, the samples are treated with a solvent that removes solely the irradiated polymer. A thin metallic film of a few nanometers is subsequently evaporated onto the sample, hence filling out the formally etched grid-like pattern. At last, the original non-irradiated polymer is dissolved, leaving onto the sample surface solely the metallic grid (for details refer to [16]). The kind of evaporated metal is chosen depending on its ductility and thermal stability with respect to the substrate. For instance, the thin film must be thermally stable at 
the experimental temperatures. It is also preferable to avoid alloying with the sample. Gold alloys with aluminum and thin gold films are easily dewetting at temperatures of $400^{\circ} \mathrm{C}$ and above [12]. Therefore, our choice came to platinum.
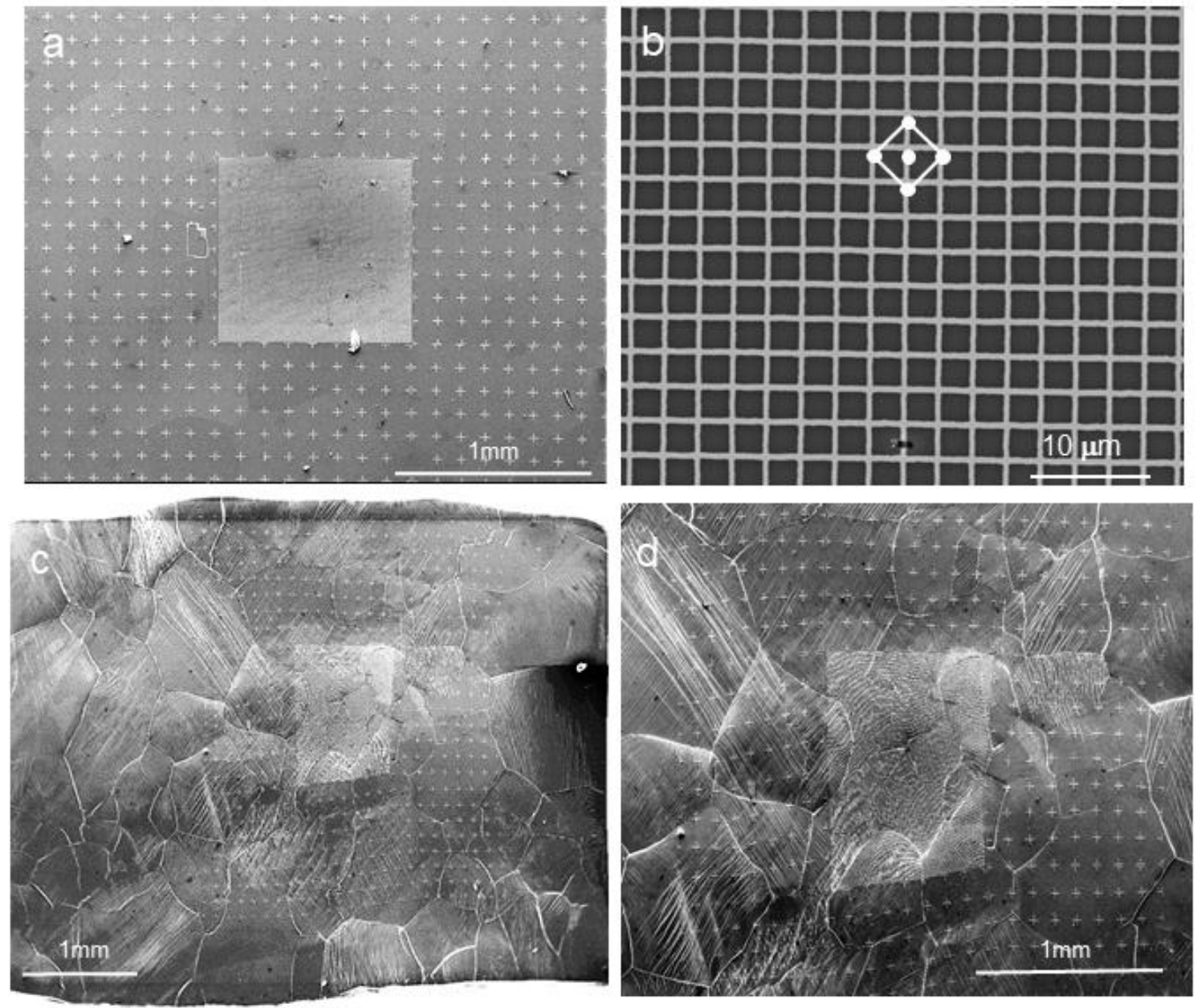

Figure 5: SEM micrographs showing the two-scale typical patterns of platinum deposit obtained by electron-microlithography (before and after deformation). a: Crosses are distant by $125 \mu \mathrm{m}$ one from another (gage length $250 \mu \mathrm{m}$ ) and cover the ROI- 2 of $3 \times 3 \mathrm{~mm} 2$. ROI- 1 covers $1 \times 1 \mathrm{~mm} 2$ in the middle of ROI-2. b: Detail of the central grid pattern of ROI-1, with $3 \mathrm{~mm}$ pitch. The pattern is used to calculate the local strain fields at the measurement points corresponding to the grid intersections. The strain computation contour is based on the nearest 4 neighbors, as schematically indicated in the middle of the image, hence resulting in a local gage length of $6 \mu \mathrm{m}$ c: Low magnification view of a whole specimen deformed at $300^{\circ} \mathrm{C}$ up to 5\% horizontal shortening. d: Central part of the specimen in (c). The crosses-marked ROI-2 allows to compute the strain field with low resolution, (but high accuracy) over a large area and to quantify the average sample shortening. The central grid-marked ROI- 1 allows to compute highly resolved full strain fields, in order to identify and quantify the deformation mechanisms.

In practice, the measurement points of the computational array can be centered at the intersections of the surficial platinum grid, as shown schematically in Figure 5b. In this present case, the chosen configuration of 4 neighbors defines a lozenge-shaped integration contour. The resulting local gage length of $6 \mu \mathrm{m}$ determines the spatial resolution of the in plane computed strain field. The Pt surficial grid pitch may be adjusted depending on the resolution wanted for the computed in plane strain field. We have selected two regions of interest. ROI-1 represents $1 \mathrm{~mm}^{2}$ centered within the sample observation surface $\left(6 \times 4 \mathrm{~mm}^{2}\right)$. ROI-2 represents $3 \times 3 \mathrm{~mm}^{2}$ surrounding ROI-1. ROI-2 excludes the sample borders that may be concerned with end effects and fretting, but remains large enough to be representative of 
the strain experienced by the specimen as a whole. The mean strain components are computed over the latter domain with a large gage length (ca $250 \mu \mathrm{m}$ ), masking fine scale heterogeneities, but ensuring computational accuracy and rapidity. Nearly real-time DIC can be performed at the mesoscopic scale over ROI-2, providing a better monitoring of the deformation process than relying on the displacement sensor of the cross-head, which measurement is affected by the compliance of the mini-rig. The pattern of the deposited platinum covering the ROI-2 represents a regular grid of crosses with a pitch of $125 \mu \mathrm{m}$ (Fig. 5), that remains substantially lower than the mean grain size (ca $300 \mu \mathrm{m}$ ). ROI-1 is selected with respect to the homogeneity of the microstructure and the absence of remaining texture. The pattern of the deposited platinum covering the ROI-1 represents a continuous regular grid. This domain is meant for detailed investigation of strain heterogeneities and active mechanisms at the scales of the individual grain and the aggregate. The grid pitch is therefore reduced down to $3 \mu \mathrm{m}$ (Fig. 5), allowing computing with fine gage lengths (ca $6 \mu \mathrm{m}$ ) and providing highly resolved strain fields. The grid lines are $600 \mathrm{~nm}$ wide, which represents a few pixels in length for the micrographs with resolution of $4096 \times 3775$ pixels.

The fine scale grid of ROI-1 shown in Figure 5 exhibits a Moiré effect (Fig. 5), due to the combination of subsampling (e-beam spot size smaller than pixel size) and slight misalignment between the marking grid and the scanning direction. The Moiré effect can be attenuated with application of a Gaussian filter during post-processing.

The grid of measurement points devoted to DIC is adapted to the different marking patterns for ROI-1 and ROI-2. For the former and latter ones the measurement points are located onto the intersections of the grid and onto the crosses, that is spaced by 12 and 171 pixels, respectively. For ROI-1, the correlation windows are 12 x 12 pixels, and hence the whole area is covered without overlapping of neighboring windows, which prevents smoothing of strain heterogeneities. The subpixel correlation is performed with a bilinear interpolation ([18], [19], [20]).

\subsection{Strain computation}

The DIC procedure delivers the displacement field of the measurement points, which allows strain computation. The integration contour we used to compute the local strain at each point is diamond shaped. It is defined by the 4 nearest neighboring points (integration scheme 2 defined in [12]). The grid of points has a pitch of $3 \mu \mathrm{m}$, therefore the local gauge length is of $6 \mu \mathrm{m}$ (see Fig. 5b).

The DIC provides only the in plane displacements and thus we can only compute the corresponding components of the in-plane transformation gradient $\mathbf{F}: \mathrm{F}_{11}, \mathrm{~F}_{22}, \mathrm{~F}_{12}, \mathrm{~F}_{21}$. In order to compute the strain tensor some assumptions are necessary in the framework of small strains. First, the normal to the observation plane is considered to be a principle 
axis of deformation and no out-of-plane rotations are considered, which implies $F_{13}=F_{31}=0$. The last hypothesis assumes axisymmetric conditions (transverse isotropy), which allows to compute the out-of-plane strain component.
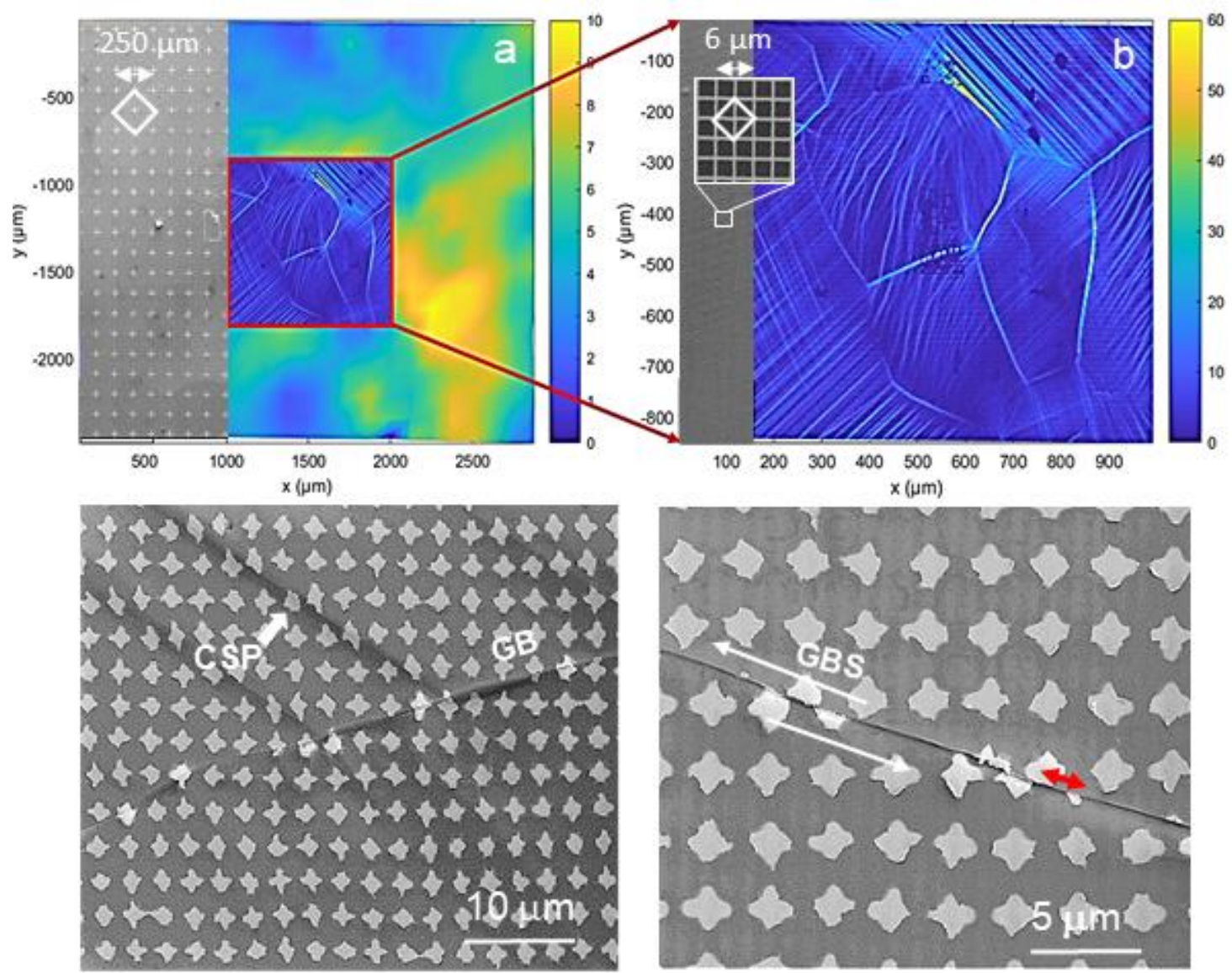

Figure 6: Computed strain maps corresponding to the ROI-2 and ROI-1 shown in Figure 5a, representing the "von Mises" equivalent strain map (in percent), for an in-situ test at $200^{\circ} \mathrm{C}$ at $5 \%$ overall axial shortening. a: The strain field of the $3 \mathrm{~mm}$ large ROI-2 is poorly resolved, owing to the rough surface marking: the array of Pt crosses shown on the left hand of the map implies a large gage length of $250 \mu \mathrm{m}$ (see text). It allows for a fast and accurate computation of the overall strain. b: The $1 \mathrm{~mm}$ large ROI-1 (centered within ROI-2) has a fine scale marking (Pt grid, shown on the left hand of the image), allowing for a gage length of $6 \mu \mathrm{m}$, and hence a highly resolved strain field showing strain localization at the grain scale. Localizations happen in a discrete manner within individual grains, due to activation of crystal slip plasticity (CSP). Crystal slip bands are shown in higher magnification in (c). Grain boundary sliding (GBS) is also evidenced by localization at some interfaces (see text). GBS is shown in (d). The red double arrow shows the offset resulting from GBS. Note that in (c) and (d) the markers are small size irregular crosses: an alternative to the regular grid.

Two examples of strain computation at the scales of ROI-1 and ROI-2 are shown in Figure 6. Their respective local strain gages are of $6 \mu \mathrm{m}$ and $250 \mu \mathrm{m}$, and hence the corresponding resolutions. The concerned test was performed at $200^{\circ} \mathrm{C}$, until axial shortening of $5 \%$. The mean axial strain component is computed in real time during the test over the whole ROI-2 domain. The strain maps presented in this work show a scalar representation of the local plastic deformation. We use the second invariant of the deviatoric part of the in-plane strain tensor, which is the 2D analog to the von Mises equivalent strain. With the above mentioned assumptions the latter may be written as $\varepsilon_{\mathrm{eq}}=2\left|\varepsilon_{1}-\varepsilon_{2}\right| / 3$, where $\varepsilon_{1}$ and $\varepsilon_{2}$ are the in-plane principal strain components. 


\subsection{Testing protocol}

A strict testing protocol was developed to ensure the reproducibility of the results. At first, the cooling circuit is stabilized at the cooling temperature corresponding to the testing high temperature, as established during the calibration procedures. Secondly, the load cell is preloaded at $100 \mathrm{~N}$, the sample is inserted in between the SiC pistons, where it is maintained with an additional preload of $10 \mathrm{~N}$.

Correct sample positioning requires the observation surface and the painted lateral facet to be perpendicular to the SEM column and to the pyrometer optical line, respectively. The pyrometer alignment is tuned by focusing its in-built LED onto the painted sample face. When vacuum reaches $10^{-3} \mathrm{~Pa}$, the sample emissivity parameter is set to the value corresponding to the desired temperature (according to our calibration, see section 2.2) and the heaters are powered. During the heating and thermal expansion phase, the crosshead position is adjusted to maintain the pre-loading of the sample around $10 \mathrm{~N}$. Equilibrium temperature is reached within less than an hour. Then the electron beam parameters are set at $15 \mathrm{kV}$ and a spot size of 4.5 .

The initial state micrographs are the references for the correlation procedures. The reference state is also used to estimate the systematic errors, trough the analysis of a known transformation, introduced by small variations of magnification.

The deformation is realized stepwise, by increments of about $0.5 \%$ axial strain, at controlled crosshead displacement rate of $0.1 \mu \mathrm{ms}^{-1}$ corresponding to a strain rate of about $10^{-5} \mathrm{~s}^{-1}$. The axial crosshead displacement is controlled by the displacement sensor, whilst the total average axial strain is monitored by a quick DIC computation, performed over the $3 \mathrm{~mm}$ - wide area (ROI-2) marked by crosses (Fig. 5 and 6). Higher magnification micrographs are further collected over the central $1 \mathrm{~mm}$ - wide ROI-1 area, marked by fine grids or dots, in order to compute later on highly resolved full strain fields (Fig. 5 and 6).

\section{Results and discussion}

The DIC-based full strain field computations which are realized over the larger $3 \mathrm{~mm}$ - wide areas (ROI-2) enable to extract precisely the total average axial strain for the central part of the specimen. The computed data and the macroscopic stress (considering applied force and actual sample cross section) are used to plot the characteristic loading curves of strength versus axial strain shown in Figure 7. The advantages of such measurements with respect to the usual macroscopic records of strain by displacement sensors are to exclude machine-related effects (emplacement the loading column and frame compliance), but also to limit the sample-scale strain heterogeneities 
related to piston fretting effects. The loading curves correspond to room temperature and three high temperatures: 110 , 200 and $300^{\circ} \mathrm{C}$, representing respectively homologous temperatures of $0.4,0.5$ and 0.6 . Yield stresses and strain hardening rates decrease monotonously with increasing temperature. These characteristic parameters observed at the macroscopic scale indicate progressive enhancement of cross slip, dislocation climb and related dynamic recovery processes, operating at the microscale. The latter will be addressed in more details in the next sections, where we focus on identification and quantification of the active deformation micro-mechanisms at the light of the kinematic analysis performed by DIC.
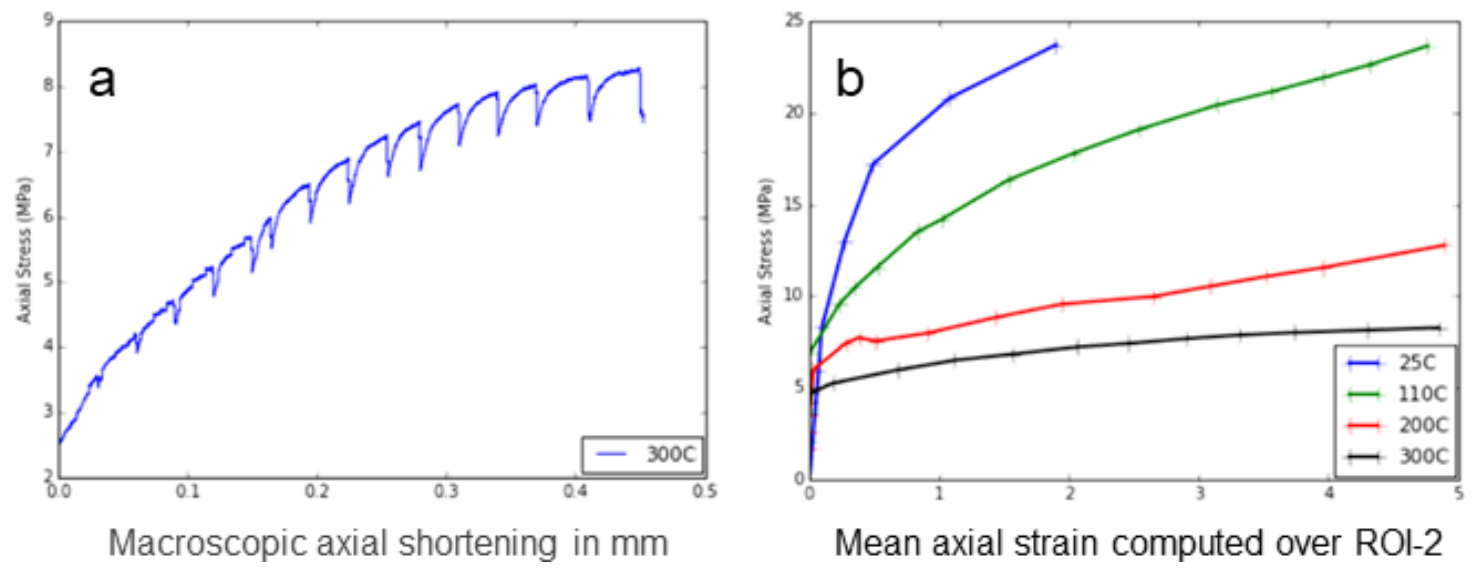

Figure 7: Mechanical curves for AlMn0.1 compressed at different temperatres. a: Raw loading curve based on the data recorded by the load cell and the linear displacement sensor attached to the mobile crosshead. b: Stress - Strain curves for $25^{\circ} \mathrm{C}, 110^{\circ} \mathrm{C}, 200^{\circ} \mathrm{C}$ and $300^{\circ} \mathrm{C}$ based on the data recorded by the load cell and the mean strain computed over the large central ROI-2 zone (see Figures 4, 5 and 6). The raw step-by-step loading curve represents the mechanical behavior of the sample combined with the compliance of the machine and the emplacement of the loading column. Conversely, the computed Stress-Strain curves represent solely the mean effective strain of the large central area of the sample (ROI-2). Note the relaxation of the material during the time laps corresponding to imaging after each loading steps.

\subsection{Influence of temperature}

Figure 8 summarizes the results of uniaxial compression tests performed at ambient $25^{\circ} \mathrm{C}, 110^{\circ} \mathrm{C}, 200^{\circ} \mathrm{C}$ and $300^{\circ} \mathrm{C}$, that is respectively $0.3 \mathrm{Tm}, 0.4 \mathrm{Tm}, 0.5 \mathrm{Tm}$ and $0.6 \mathrm{Tm}$. Figures $8 \mathrm{a}$ to $8 \mathrm{~d}$ presents the equivalent von Mises strain maps computed by DIC over the central $1 \mathrm{~mm}$-wide area (ROI-1) for the final deformation step, cumulating axial strains of c.a. $2 \%$ for room temperature and c.a. $5 \%$ for higher temperatures. Note that the color scale, given in strain $\%$, is not the same for all the tests. Figures $8 \mathrm{e}$ and $8 \mathrm{f}$ show the Z-contrast and secondary electrons micrographs corresponding respectively to the original microstructure and the deformed state (with marker grid) of the specimen deformed at $110^{\circ} \mathrm{C}$. Both the SE micrographs and the DIC-computed equivalent strain maps allow to see the activation of crystal slip, which results in the occurrence of surface slip lines in the individual grains. Yet, both types of observations do not always exhibit the same slip lines. The SE imaging evidences the development of topography which is essentially related to the out-of-plane component of active crystal slip systems. We will call the latter "physical slip lines" (PSL), because of the remaining relief. Conversely, the DIC-computed strain maps inherently 
evidence solely the in-plane slip components of active slip systems. When both components are present, both observations reveal the same slip lines. But, in extreme cases, when the out-of-plane component of a slip system is negligible, the slip lines are evidenced solely by the DIC-computing procedure. We will call the corresponding slip lines "kinematic slip lines" (KSL), as they do not leave any remaining physical signature. In fine, the two observation techniques are complementary for the purpose of detailed investigations of plastic strain heterogeneities.
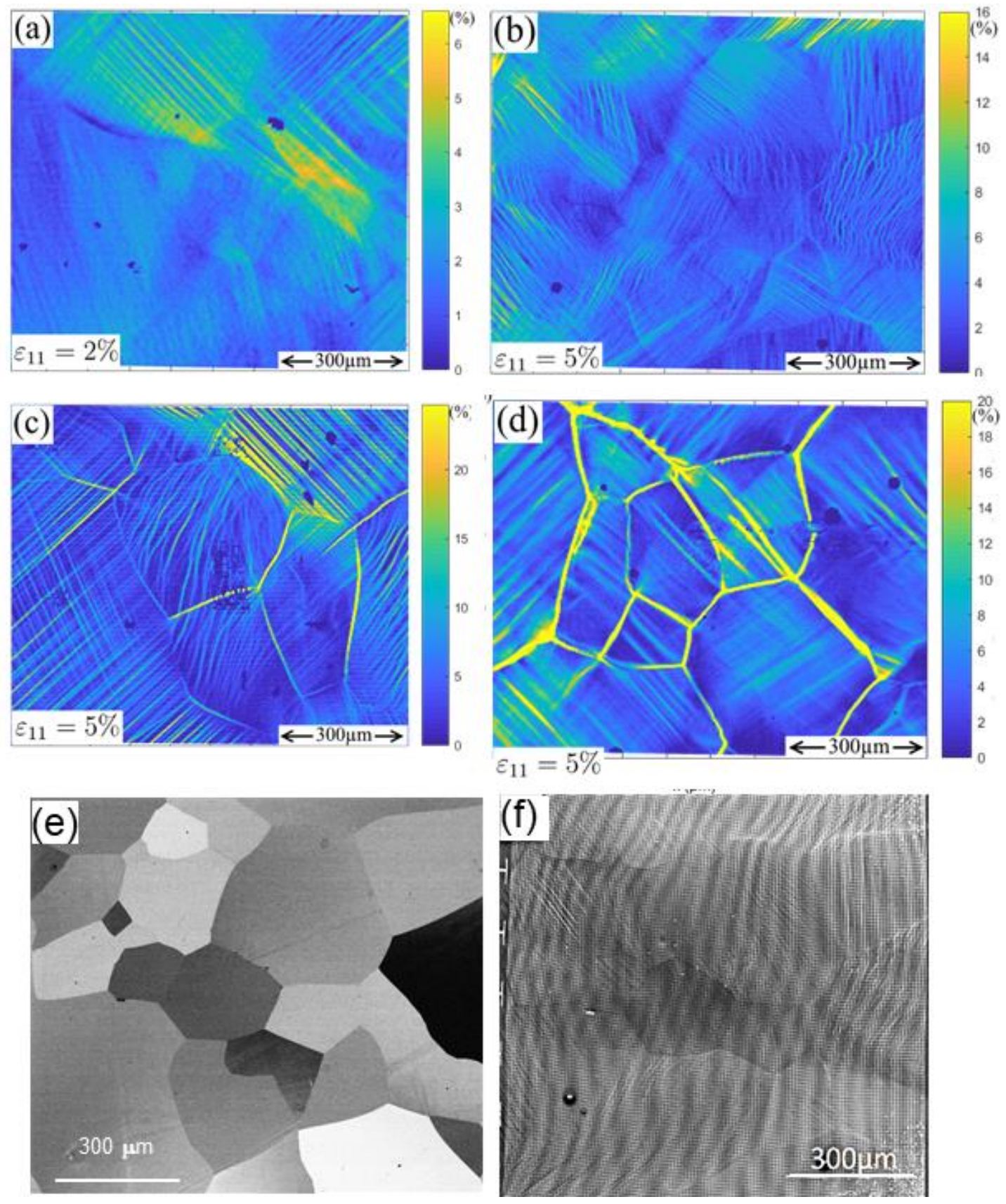

Figure 8: Typical computed strain maps and SEM micrographs of compression tests at ambient temperature and $2 \%$ axial strain (a), $0.4 \mathrm{Tm}, 0.5 \mathrm{Tm}$ and $0.6 \mathrm{Tm}$ and $5 \%$ axial strain (b to d, respectively). Note, the strain maps (a to c) are not at the same color scale. e and f correspond respectively to SEM micrographs of the initial microstructure (Z-contrast, without marker grid) and the deformed state (SE contrast, with marker grid) of the sample compressed at $0.4 \mathrm{Tm}\left(110^{\circ} \mathrm{C}\right)$. 
The spatial resolution of the strain field mapping is however limited by the micrometer-sized local strain gage, whilst the SEM micrographs have a spatial resolution of about $120 \mathrm{~nm}$. In order to obtain finer strain field resolution, we have developed a technique consisting in dewetting thin metallic films ([12], [24]). The latter are deposited by classical thermal evaporation or plasma sputtering onto the polished sample substrates with thicknesses ranging between a few $\mathrm{nm}$ to a few tens of $\mathrm{nm}$. Annealing the coated samples at temperatures above $350^{\circ} \mathrm{C}$ leads progressively to cavitation, followed by development of dendritic patterns. The final stage of the dewetting process results in the formation of metallic quasi-spheres with sizes of a few tens of nm to a few micrometers, depending on the starting film thickness, the annealing temperature and time. But, this technique is most appropriate to the study of very fine grained materials and was therefore not applied in the present study.

The DIC-computed strain maps for samples deformed up to ca $5 \%$ at 110,200 and $300^{\circ} \mathrm{C}$ clearly evidence major differences of crystal slip and localization patterns. At moderate and intermediate temperatures $\left(110^{\circ} \mathrm{C}\right.$ and $200^{\circ} \mathrm{C}$, respectively) we can see some similarities and some differences. At first, it is worthy to note that in both cases slip lines are essentially sharp. At moderate temperature the spatial density of slip lines is high, but their strain intensity is low, and little localization of strain is detected at grain boundaries (Fig. 8a). At intermediate temperature $\left(200^{\circ} \mathrm{C}\right)$ strain intensity of individual slip lines increases, whilst their spatial density decreases. Strong localization appears along several grain boundaries (Fig. 8b). At moderate temperature most of grains present both wavy and straight slip morphology, whilst the latter dominates at intermediate temperatures. At higher temperature $\left(300^{\circ} \mathrm{C}, \mathrm{Fig} .8 \mathrm{c}\right)$ slip lines present mostly straight but diffuse character. They broaden considerably, but lose their sharpness. These morphological features may be related to recovery processes which are increasingly active with temperature. At room temperature crystal plasticity occurs by easy octahedral slip. At moderate temperatures $\left(100-200^{\circ} \mathrm{C}\right)$ are active both easy octahedral slip and non-octahedral, or cross slip. Thermally activated non octahedral slip in FCC metals is reported in literature ([25], [26], [27], [28], [29]) and is evidenced in the present work by the appearance of wavy slip lines, as shown in Figure 8. At moderate temperatures the kinetics of recovery processes related to dislocation climb (formation of dislocation walls and sub-grain boundaries) are low. Dislocation densities readily increase. Mutual pinning of gliding dislocations from different systems results in entanglements (forest effects), and hence pronounced strain hardening (Fig. 7). The latter is however moderated by spreading crystal slip: that is the activation of numerous slip bands, carrying limited slip amounts. Conversely, at higher temperatures $\left(300^{\circ} \mathrm{C}\right)$ dislocation climb processes are active, thus allowing for recovery and limiting strain hardening (Fig. 7). Therefore, crystal slip may be efficiently localized within fewer slip bands. Very similar recent observations are reported for $\mathrm{NaCl}$ single crystals deformed at room and high temperature ([30]). Most importantly, at high temperatures $\left(300^{\circ} \mathrm{C}\right)$ all grain boundaries present very 
strong localization. The latter is due to grain boundary sliding (GBS), which presents both large in-plane component as evidenced by the micrographs in Figure 9, and important out-of-plane component as evidenced by local AFM measurements (Fig. 9). The latter induces the development of strong topography, and in some cases grain thrusting. In this case the surface marking pattern is partly lost due to the overlapping. In other cases, the in-plane GBS component is so important that marking patterns are extremely deformed and damaged. For these reasons, the DIC computation of strain along grain boundaries obtained at $300^{\circ} \mathrm{C}$ might be considered only as a lower bound. It is worth noting that GBS is usually invoked for superplastic behavior and is mostly associated with small grain sizes of the order of a few tens of $\mu \mathrm{m}$ or less ([6], [31], [32], [33], [34]). In the present study we show that GBS is clearly active even for a coarse microstructure with grain sizes of several hundreds of $\mu \mathrm{m}$. A recent study of in situ SEM compression of coarse grained aluminum also evidenced active GBS ([10]). A previous study based on DIC analysis of in situ SEM compression testing of synthetic rock salt have shown that GBS contribution to the overall strain increased with decreasing grain size ([12]). For coarse microstructures comparable to ours GBS contribution to the overall strain of rock salt was estimated to be of less than $10 \%$ ([12], [13]). However, for rock salt deformed at room temperature, GBS involves grain boundary decohesion, frictional sliding, micro-damage and dilation, whilst in our case GBS is fully ductile. Quantitative analysis of GBS in the present samples deformed at $200^{\circ} \mathrm{C}$ will be addressed elsewhere.

An additional complication at the higher temperature relates to grain boundary (GB) migration. The latter may be a high temperature recovery mechanism, participating for instance to the dynamic recrystallization of highly strained polycrystals during hot-working. In this case, the interface between two grains may migrate at the expense of one grain, under the action of driving forces arising from the difference of free energy related to different plastic strains and the resulting difference in elastic strain energy on both sides of the boundary. Usually, marked GB migration occurs at high temperatures and strains and induces very strong evolution of the starting microstructure, which precludes kinematic analysis by DIC. In our case (low strain) GB migration is not observed up to $200^{\circ} \mathrm{C}$. It happens modestly at $300^{\circ}$, but starts to be more pronounced at $400^{\circ} \mathrm{C}$. Curiously, GB migration does not affect the surface marking grid, which remains preserved during the process. The latter observation suggests the process to be essentially diffusive, and thus beyond the continuum mechanics scale. Yet, it complicates DIC computation, because of the progressive loss of correspondence between the preserved markers and the diffusively evolving microstructure. When considering the cumulative strain with respect to the initial state as reference, GB migration results in artificially spread localization along the migrating portions of sliding grain boundaries, as shown in Figures $8 \mathrm{~d}, 9 \mathrm{c}$ and 10 , Though, the kinematic analysis remains possible, when considering solely the previous step of loading as reference. Conversely, GB migration may also operate at small scales and strains, being coupled with limited relative grain displacement, 
which is known as shear coupled GB migration. All the experimental and numerical studies addressing this mechanism are based on TEM observations and atomistic scale simulations of particular grain boundaries. The latter phenomenon relates to specific grain boundary defects called disconnections and is also operational at low temperature ([35], [36], [37], [38]). While being an important phenomenon involved in dynamic recrystallization and grain growth processes in polycrystalline materials, GB migration is beyond the scope of this study and we will not elaborate further on it.
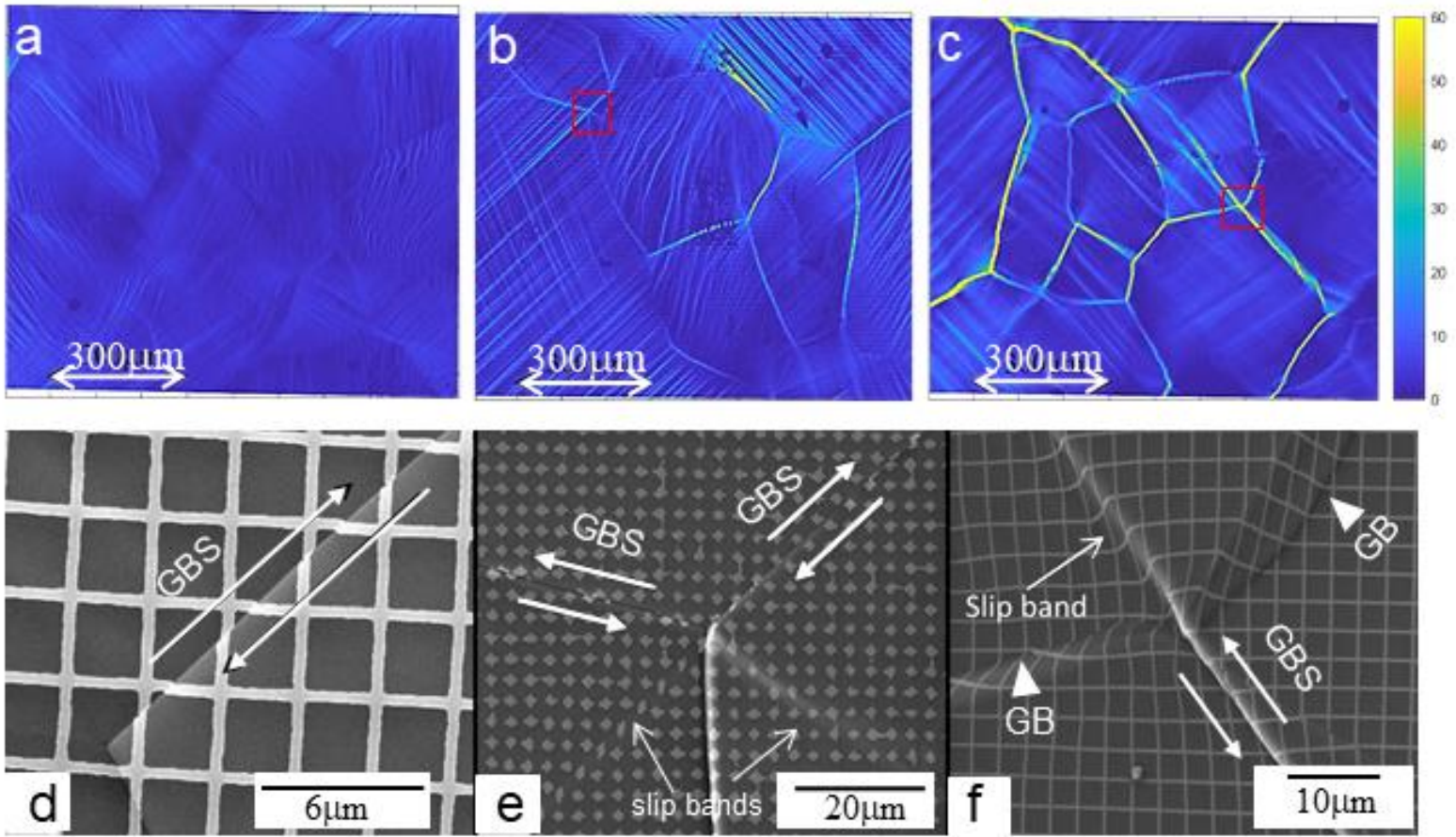

Figure 9: a, b and c: Same strain maps as in Figure 8 (for samples deformed at $0.4,0.5$ and 0.6 Tm up to $5 \%$ axial strain), but shown to the same color scale. It is evidenced that increasing temperature results in lower spatial density of slip lines, but in higher intensity of slip (see text), which means higher localization. Grain boundary sliding is also strongly enhanced by temperature. d, e and f: SE mode micrographs of selected triple junctions, respectively from the same specimens as in a, b and c. At $0.4 \mathrm{Tm}$ it is evidenced little grain boundary sliding. At higher temperature, this mechanism is accompanied by intense crystal slip activity within the third grain, which is facing the sliding grain boundary and acts as an incompatibility for GBS. The orientation of the third grain will strongly impact the GBS ability.

A previous study based on DIC analysis of GBS and CSP in synthetic rock salt ([12]) has shown that GBS and CSP mechanisms are concomitant and interactive, each one being an accommodation mechanism for the other. GBS was activated along boundaries where occurred plastic incompatibilities between neighboring grains. Conversely, GBS activation was accommodated at the triple junction by localized CSP in the third grain, facing the sliding interface. A second study of ductile deformation of rock salt ([13]), based on both 2D DIC and 3D DVC (digital volume correlation) techniques have shown that at the experimental room temperature conditions the GBS phenomenon relates to grain boundary decohesion and frictional sliding, resulting in diffuse micro-damage, as evidenced by the computed 
dilatant volumetric strain. While in the present case the material deforms wholly plastically, we do observe similar phenomena of interacting GBS and CSP, as shown in Figures 9 and 11.
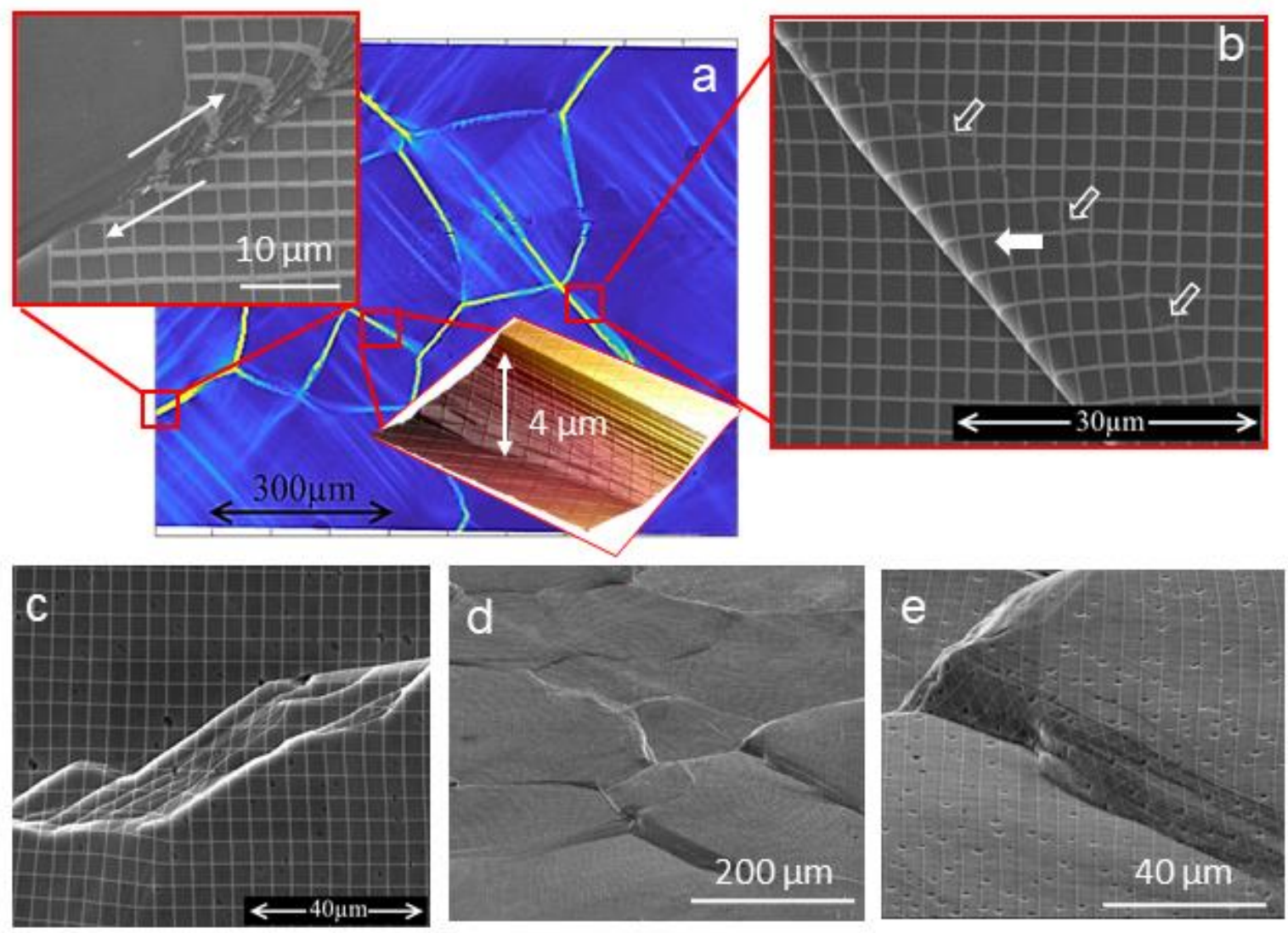

Figure 10: Intense GBS at $300^{\circ} \mathrm{C} \mathrm{(a} \mathrm{and} \mathrm{b)} \mathrm{and} 400^{\circ} \mathrm{C}(\mathrm{c}, \mathrm{d}, \mathrm{e})$ leads to development of marked topography (a, $\mathrm{d}$ and e) and grain boundary migration (b and c). Above $300^{\circ} \mathrm{C}$ the GBS is so active that the marking grid may be severely distorted/damaged (a). Continuous (b) or step-wise (c) grain boundary migration occurs, leading to substantial local microstructural modification of the interfaces. Very important out of plane grain sliding and grain overlapping, clearly evidenced by observing tilted samples (d, e), lead to damage and/or to loss of marker grid. All these phenomena may substantially complicate DIC computation (see text).

From a phenomenological view point, in hot-deformed fine grained materials GBS may be considered as the dominant deformation mechanism per se, in which case local CSP, GB migration and/or diffusional mass transfer act as local accommodation mechanisms at multiple grain junctions. Conversely, for coarse grained materials dominated by CSP, GBS may be seen as a necessary accommodation mechanism across interfaces where CSP transmission from a grain to another is inhibited due to crystallographic incompatibilities. Thought, in the latter case the phenomenology of GBS may rely on different microphysical mechanisms active along GBs, including diffusional transfer, dislocation activity, or micro-damage and frictional sliding. 


\subsection{Strain localization evolution}

DIC analysis offers the opportunity to follow either the cumulative evolution of strain localization in reference to the initial state, or the incremental evolution by referring to each previous loading step. Figures 10a and 10b show the central ROI-1 with surface marking before and after deformation at $200^{\circ} \mathrm{C}$. Some grains are labelled for the purpose of discussion. The Figures 10c to 10f show the cumulative development of strain localization at loading steps 2, 4, 7 and 10 corresponding respectively to the yielding point and c.a. $0,5 \%, 1.5 \%, 3 \%$ and $5 \%$ shortening. Interestingly, we can see in Figure 10c (step 2) that the early strain localization occurring right after yielding corresponds simultaneously to both crystal slip and GBS: the upward and downward arrows points respectively to a slip band and GBS. Yet, at this very early stage of deformation localization appears mostly in favorably oriented grains like $g_{1}, g_{4}, g_{5}$ or $g_{6}$. The latter activate only single octahedral slip (strait slip bands), as evidenced by combined EBSD and geometrical analysis of slip traces (which will be discussed elsewhere). At about $1.5 \%$ axial strain (step 4), localization patterns clearly affect all of the grains, combining CSP and GBS. At this stage, unfavorably oriented grains like $\mathrm{g}_{2}, \mathrm{~g}_{3}$ and $\mathrm{g}_{7}$ readily activate non-octahedral cross-slip (wavy slip bands denoted by a horizontal arrow). In overall, one can see in Figures $10 \mathrm{e}$ and $10 \mathrm{f}$ that the density of slip bands and their activity progressively increase with deformation (c.a. $3 \%$ and $5 \%$ shortening, respectively). In more details, it appears that the favorably oriented grains activate double octahedral slip, whilst the non-octahedral slip intensifies within unfavorably oriented grains. It is noteworthy that plastic compatibility between neighboring grains is sometimes ensured by both transmission of crystal slip across grain boundaries and by GBS, as indicated by the NE-SW oriented solid arrows. When an apparently direct continuity of slip bands exists across interfaces, as for grains $\mathrm{g}_{4}$ and $\mathrm{g}_{5}$ (indicated by the NE-SW oriented hollow arrow), the GBS component is low. When the neighboring grains present stronger plastic incompatibility, as for grains $g_{2}$ and $g_{6}$ (indicated by the NW-SE oriented hollow arrow), the GBS component is high.

Localized intense slip bands originate from many triple junctions facing sliding grain boundaries (solid arrows in Fig. 10f). Arrows labelled 1 and 2 point to triple junctions where two slip bands originate, each facing an adjacent sliding grain boundary. Arrows labeled 3 and 4 point to triple junctions where single slip bands originate. It is not always clear weather a CSP band emerging at a triple junction activates GBS along the facing grain boundary, or if it operates the other way round, the active GBS between two grains triggering a CSP band starting from the triple junction within the facing third grain. It is likely both cases may happen. However, considering in Figures 10c to 10f the evolution of length and intensity of the slip band pointed by the arrow 4 the second option seems favored. In any case, it is clear that CSP and GBS are not operating independently. 

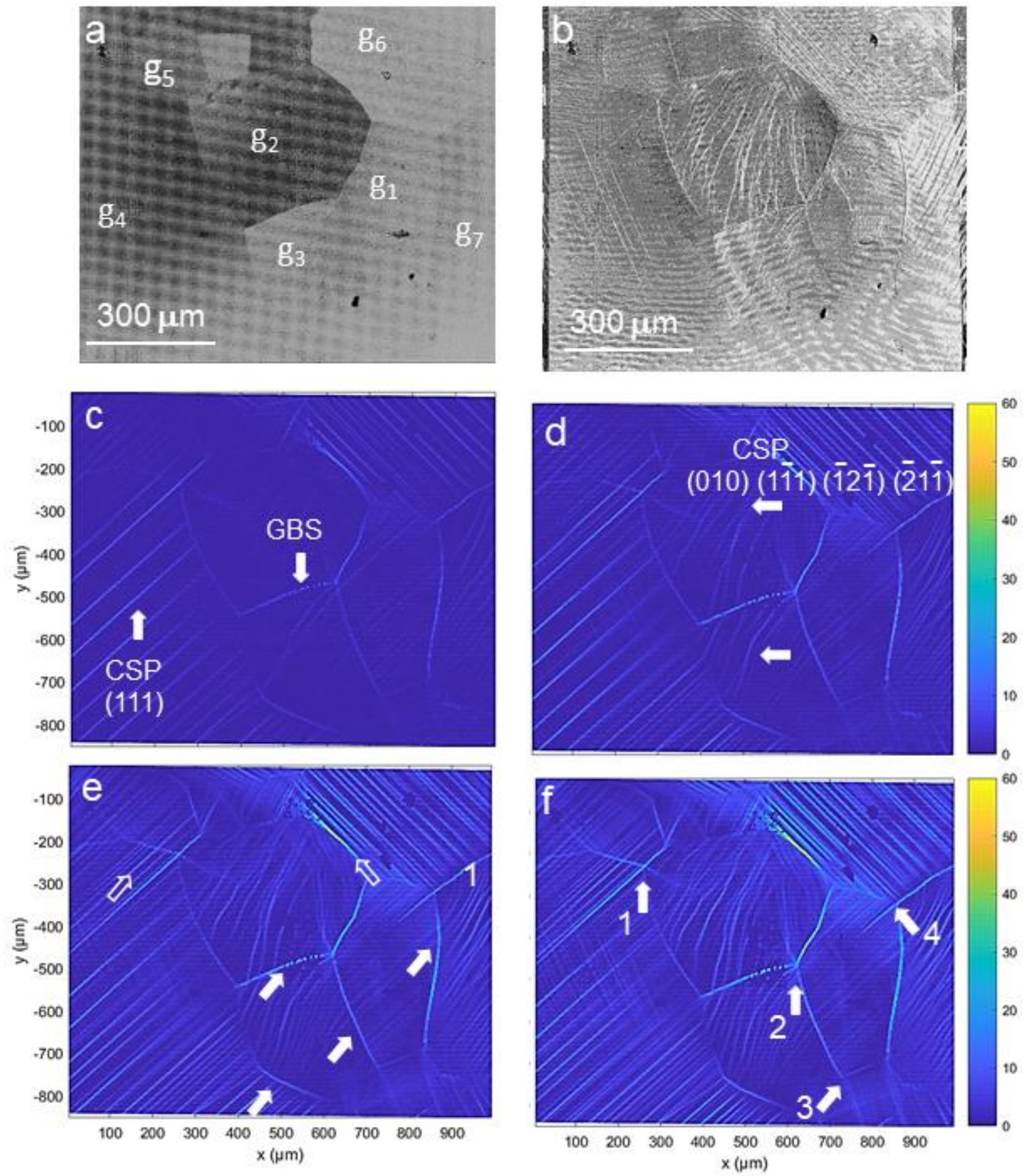

Figure 11. a: A selected ROI-1 is observed in Z-contrast mode, showing the initial microstructure. B: The same microstructure is shown in SE mode after compression at $200^{\circ} \mathrm{C}(0.5 \mathrm{Tm})$ up to $5 \%$ global shortening. The enhanced image contrast evidences the physical slip lines. c, d, e and f: Equivalent strain maps corresponding to the same ROI-1 at deformation steps 1, 4, 7 and 10 (respectively c.a. 0.5, 1.5, 3 and $5 \%$ axial strain), with respect to the initial reference image (cumulative strain fields). It is observed that both CSP and GBS activate right after yielding. The activity of both mechanisms intensify with time. Easy octahedral slip is activated first (c, straight slip lines), followed by cross-slip as evidenced by the wavy slip lines ( $\mathrm{d}$ to f). Crystal slip transmits across several grain boundaries (apparent continuity of slip bands). The intensity of the GBS component relates to the ease of crystal slip transmission. 
It is noteworthy that GBS activity is heterogeneous from a sliding interface to another. But, variation of GBS amplitude along the same interface is also observed, with maximum intensity in the central part of the grain boundary. Gradients of GBS activity were previously considered addressing strain compatibility in polycrystals [39] and were more recently reported for pure aluminum with columnar microstructure [10].
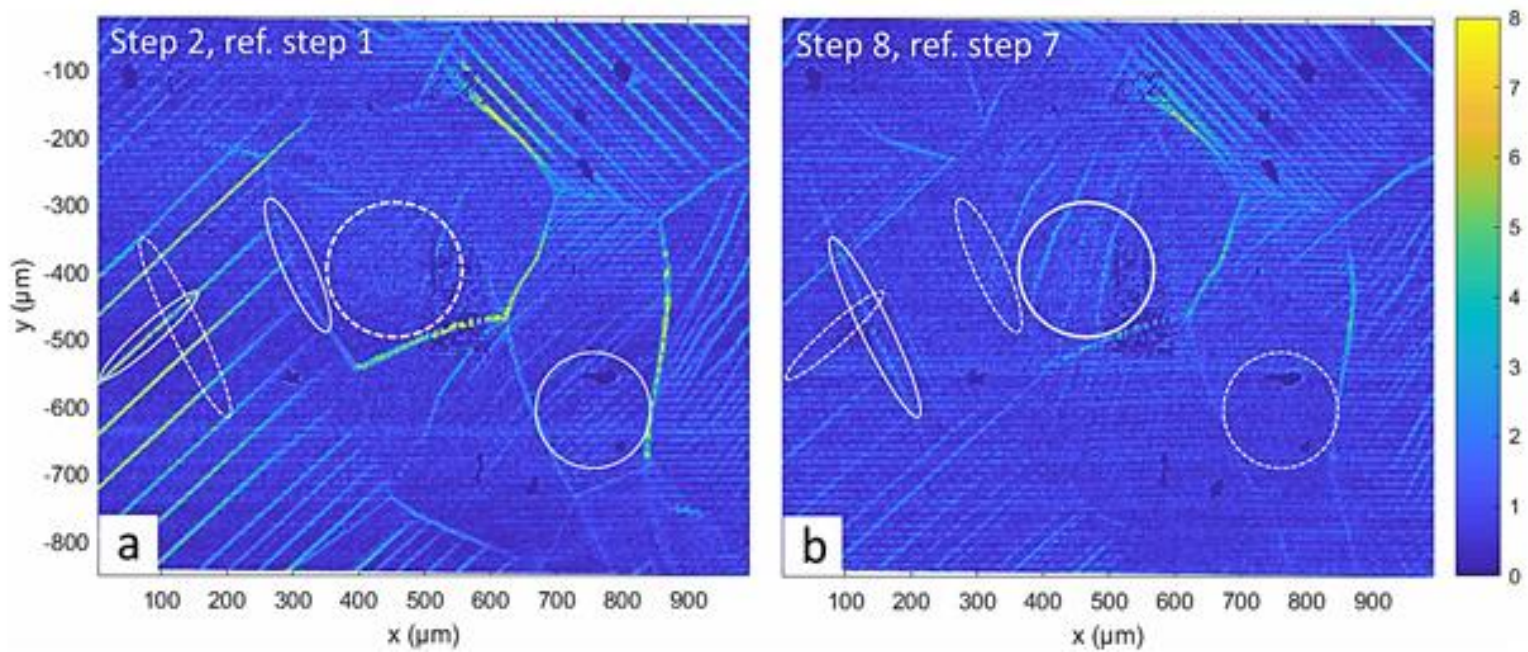

Figure 11: Strain maps for two steps of compression at $200{ }^{\circ} \mathrm{C}$ ( $\mathrm{a}$ and b) with their previous step of deformation as reference. The white ellipses highlight the slip bands and the grain boundaries that slide, which appear and disappear on strain maps during the deformation. (a) Step at $0.5 \%$ of axial strain, increment of $0.4 \%$ from the previous (reference) step. (b) Step at 3\% of axial strain, increment $0.44 \%$ from the previous step.

The incremental evolution of strain localization obtained by DIC in reference to each previous loading step is shown in Figure 11 (note different color scale than in Fig. 10). This approach allows to have a better representation of time resolved evolution of localization and hence of mechanisms activities and interactions. It is observed that the activity of crystal slip is discontinuous with time. The intensity of localization of slip bands is not monotonously increasing with loading. For instance, some previously activated slip bands may have periods of inactivity and reactivate later on. Although less pronounced, similar conclusion may be drawn for GBS too. Both mechanisms present history dependent activities. For example, in Figure 11 the solid and dashed line ellipses show respectively the emplacements of intermittent localization for both GBS and CSP, the dashed line ellipses denoting the lack of activity previously indicated by the solid line ellipses. The final picture exhibited in Figure 10, shows several grains with nearly homogeneous distribution of slip lines, which carry nearly homogeneous crystal slip. The sequential evolution shown in Figure 11 demonstrates that both phenomena present substantial fluctuations along the loading history. For instance, it appears that once easy octahedral slip activates the resulting slip lines extend nearly over the whole grain, whilst the slip traces of non-octahedral cross-slip extend progressively all along the loading process. 


\subsection{Subsurface microstructure investigated by FIB and TEM}
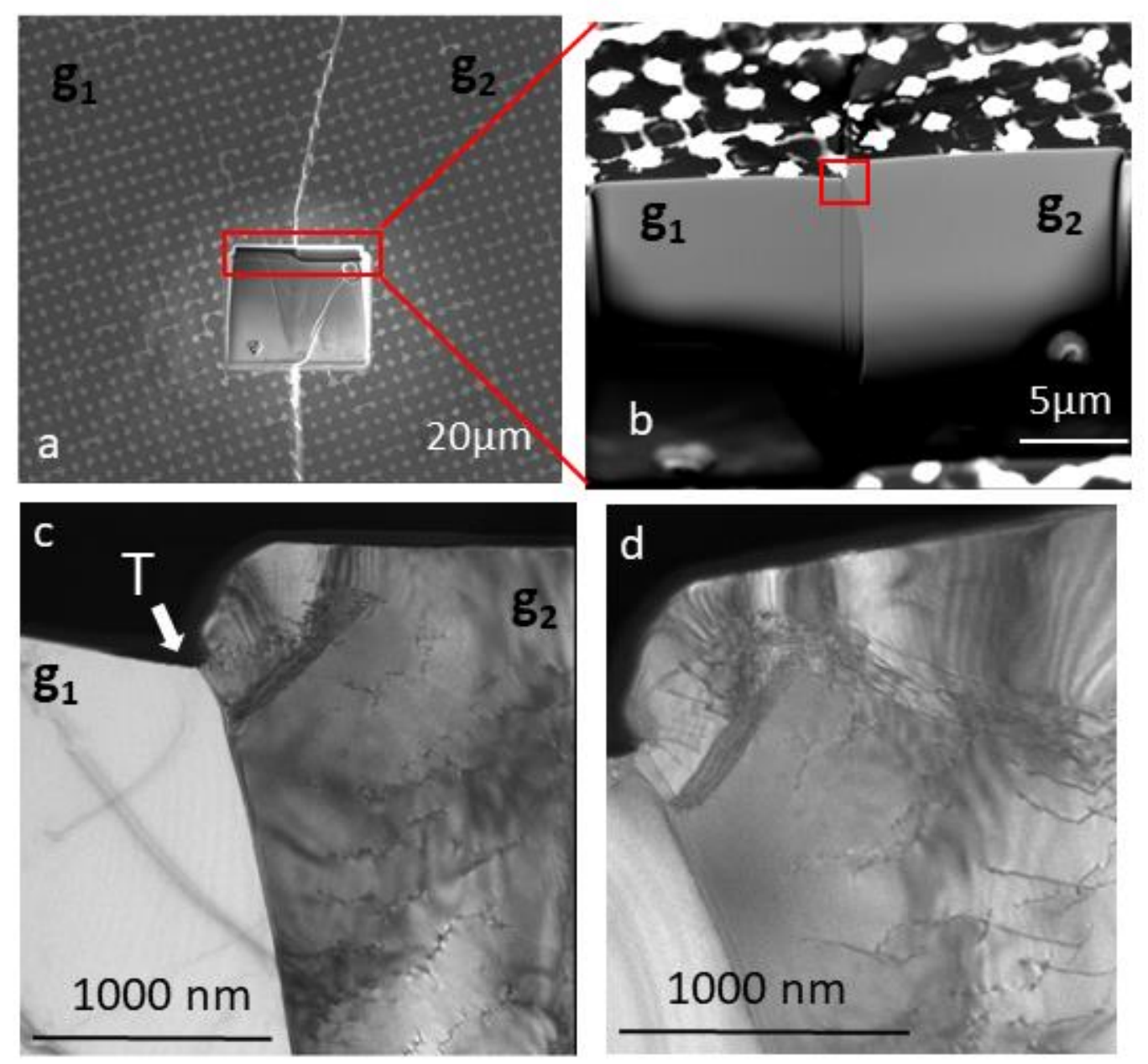

Fig. 12: a: Same sample as shown in Fig. 10 and 11. Interface between grains g1 and g2 observed in the dual beam SEM-FIB (the marker pattern is not a grid, but regularly spaced micrometer-sized spots). The excavation at the central part of the interface is operated by FIB sputtering. b: Imaging of the cross section of the grain boundary from aside (tilted specimen). c and d: Two TEM bright field observations of a differently tilted cross section of the grain boundary corresponding to the red frame in (b), after machining and thinned by FIB. Grain g1, which is favorably oriented (Fig. 10 and 11) for easy octahedral slip, appears free of crystal defects in the vicinity of the grain boundary. Conversely, g2 is unfavorably oriented (see Fig. 10 and 11) and activates both octahedral and non-octahedral cross slip. It shows substantially important dislocation microstructures: free dislocations, entangled dislocations and dislocation arrays. The surficial point $\mathrm{T}$ is a source of free dislocations gliding in grain $\mathrm{g} 2$. In depth of $\mathrm{T}$, g2 is building a sub-grain boundary.

The major disadvantage of in situ SEM kinematic analysis is that the observations are surficial, which may lead to misinterpretations with respect to the real 3D microstructure. EBSD allows to precisely identify the active slip systems on the basis of surficial slip lines and the crystallographic orientation of the individual grains. Therefore, considering the macroscopic stress state, resolved shear stresses may be precisely attributed to the active slip systems. 
Conversely, similar geometrical analysis of GBS are difficult because the inclination of the GB plane in depth is unknown. For instance, the observation of measurable out plane sliding components clearly indicate that GBs may be inclined at depth, which precludes determining a resolved shear stress. It is therefore absolutely necessary to access to the real 3D microstructure of the material. These limitations of $2 \mathrm{D}$ observations could be circumvented using sophisticated 3D techniques such as DCT (diffraction contrast tomography). These are real achievements, which are however not easy to apply, because of the need to access synchrotron radiation. Furthermore, their poorer spatial resolution in comparison with SEM and TEM preclude the identification of structural crystallographic defects along grain boundaries that may be involved in the GBS phenomenon. We therefore tried to capture some aspects of sliding interfaces using FIB (focused ion beam) machining and post mortem TEM investigations, in order to characterize GB's morphology at depth and dislocation microstructures in the vicinity of GB's, respectively.

At first we used an FIB Helios-660 (at Ecole Centrale-Supelec) in order to excavate a small volume of about $100 \mu \mathrm{m}^{3}$ around GBs of interest, where GBS was identified and quantified (Figure 12a). The excavation allows to reveal the geometry of the GB in depth, and in particular its inclination, so that we could characterize the resolved shear stress into the GB plane with respect to the uniaxial loading direction. We observed that GBs presenting essentially in-plane sliding component exhibit nearly straight vertically inclined interfacial planes, as shown in Figure 12b. Secondly, we excavated a lamella of $\sim 20 \mu \mathrm{m}$ long, $\sim 10 \mu \mathrm{m}$ large and $\sim 2 \mu \mathrm{m}$ thick across the chosen GB. A platinum film was deposited on the surficial top edge of this cross section (the top of grain g1 and grain g2, see Fig. 12b), in order to protect the surface line of the GB. At last, the lamella slice was further thinned, so that the surface edge becomes transparent to electrons in the TEM.

Once mounted on the TEM copper holder, the lamella has been observed with a Titan-Femis $300 \mathrm{kV}$ microscope at the CIMEX platform. In Figures 12 and 12 we show TEM images of two lamella slices taken across selected grain boundaries. One is a random-type interface taken from an annealed undeformed aluminum, which serves as reference to the structural defects along grain boundaries (Fig. 13). The second is taken from the interface between grains g1 and g2 of a sample deformed at $200^{\circ} \mathrm{C}$ up to 5\% shortening, shown in Figures 10 and 11 (Fig. 12 and 13). The reference lamella displays very few extended crystal defects - whether there are actually none, or because of the limited thickness. Conversely, the same type of lamella from the deformed specimen shows a completely different situation in the zone shown on the red frame in Figure12b. Focusing in this zone, it appears that a sub-grain boundary seems to be under construction, and the line orthogonal to the surface of the TEM sample passing through point $\mathrm{T}$ (a kind of equivalent to a triple junction between the two grains and the free surface) may have been a source of dislocations for the building of the sub-grain boundary. Tilting the specimen slice and switching to dark field imaging 
allows to demonstrate that the sub-grain boundary is composed of two sets of parallel dislocations (Fig. 13). The mechanism allowing for GBS still remains unclear. However, it is noteworthy that g1, which exhibits double easy octahedral slip (Fig. 10 and 11) does not present remaining free dislocations or dislocation arrays. The easily gliding dislocations may have been easily annihilated when reaching the free surface. Conversely, substantial signatures of the dislocation activity is evidenced in g2 (Fig. 13), which activated both octahedral and npn-octahedral cross slip (Fig. 10 and 11). These observations suggest that $\mathrm{g} 1$ and $\mathrm{g} 2$ might have presented substantial plastic incompatibility, which might in turns be the reason for activation of GBS between these neighboring grains.

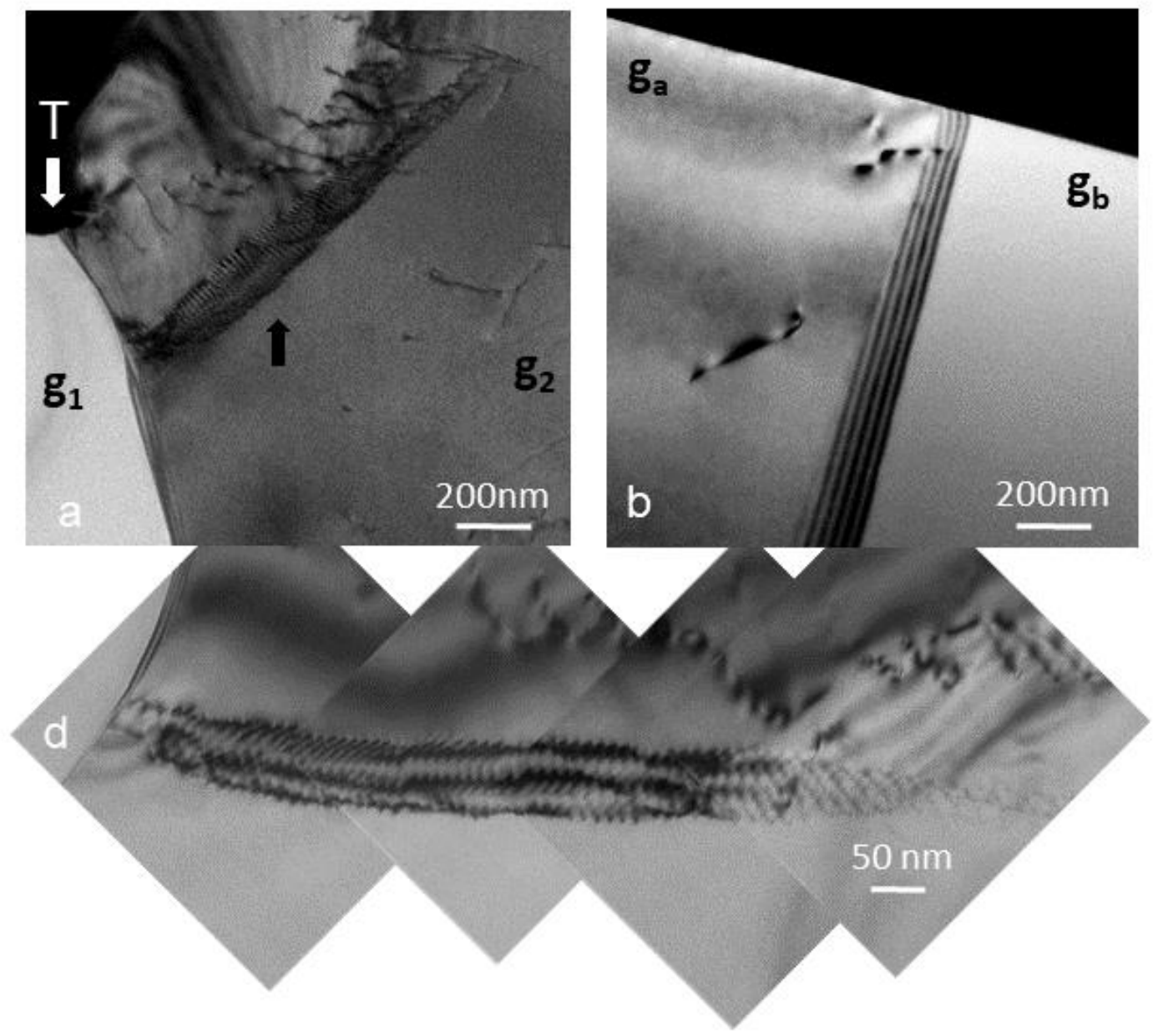

Fig. 13: a and d: Higher magnification TEM micrographs from the same slice as in Figure 12 (bright field mode). The sub-grain in g2 (black solid arrow) is shown in more details, evidencing the constitutive two sets of dislocation families. The T source may have been at the origin of the deeper dislocation array as one of the structuring set of dislocations corresponds to the free ones. b: TEM micrographs from an undeformed specimen (bright field mode). The sample is not oriented edge-on the grain boundary, showing fringes indicative of the slice thickness. Most important, very few dislocations are found in the starting type of undeformed sample.

It is admitted now that specific dislocation mechanisms occur at grain boundaries and movements of dislocations inside the grain boundaries have been observed. Apart from movement of single dislocations, we should 
also consider more complex assemblies, such as pileups at the grain boundary, which have been pointed out by DDD studies coupled to Laue micro-diffraction experiments ([40]). Our micrographs drive at exploring more boundaries, which can be an incentive to envisage additional dislocation microstructures at grain boundaries.

\section{Conclusion}

The original setup presented in this work allowed to perform multi-scale full mechanical field measurements insitu SEM, coupling hot compression tests and DIC analysis. Detailed analysis of the localization patterns allowed to discriminate the deformation mechanisms activated during viscoplastic flow of coarse grained aluminum specimens at room conditions and temperatures of up to $400^{\circ} \mathrm{C}$, and at constant displacement loading of about $0.1 \mu \mathrm{ms}^{-1}$ corresponding to a strain rate of about $10^{-5} \mathrm{~s}^{-1}$. Deformation was largely dominated by crystal plasticity, starting mostly with octahedral easy slip at room temperature, then progressively activating non-octahedral cross slip with increasing temperature. Localization intensity of slip increases with temperature, whilst its spatial distribution decreases, which suggests strong thermal healing and recovery effects. Concomitantly, minor component of GBS activates above $100^{\circ} \mathrm{C}$ and progressively increases with temperature. Above $300^{\circ} \mathrm{C}$ GBS becomes very pronounced and induces strong outof-plane grain sliding resulting in strong topography and grain thrusting. Similarly, grain migration phenomena become noticeable. Both phenomena limit the possibility of kinematic analysis by DIC. Most importantly, GBS and CSP activate at the same time, right after yielding, at axial strains of less than half a percent. GBS intensity seems to correlate with crystal slip incompatibility between neighboring grains. The latter observations indicate that for coarse grained aluminum GBS is a secondary but necessary mechanisms, allowing to accommodate local plastic incompatibilities. These observations are further supported by TEM investigations of dislocation microstructures along sliding grain boundaries. Conversely, severely localized CSP starts from triple junctions and extends within grains facing sliding boundaries. The net result is a continuous interplay between the two mechanisms, allowing the achievement of macroscopically homogeneous viscoplastic flow.

\section{Acknowledgments}

This work has been supported by the Fondation EDF (sponsoring the Chaire "Energies durables" at Ecole polytechnique) under the supervision of F. Carré. We thank V. de Greef, E. Guimbretière, H. Gharbi and J. C. Eytard for their precious technical support. We also warmly thank R. Quey (Lab. George Friedel, Ecole des Mines de Saint 
Etienne) for gently providing the aluminum alloy and Eva Héripré (MSSMAT, Ecole CenaleSupelec) for access and help with the FIB.

\section{References}

[1], Ashby, F. (1972), A first report on deformation-mechanisms maps. Acta Metallurgica, Vol. 20 (7), 887-897.

[2], Ashby, F. (1982), Mechanisms of deformation and fracture. Adv. Applied Mechanics, Vol. 23, $117-177$.

[2], H. J. Frost and M. F. Ashbv. (1982) "Deformation Mechanism. Maps: he Plasticity and Creep of Metals and Ceramics. Oxford [Oxfordshire] ; New York : Pergamon Press, 166 pp.

[4], Weertman, J. (1957) Steady-state creep of crystals. Journal of Applied Physics, Vol. 28 (10), 1185-1189 $10.1063 / 1.1722604$

[5], Weertman, J. (1957) Steady-state creep through dislocation climb. Journal of Applied Physics, Vol. 28,362 https://doi.org/10.1063/1.1722747

[6], Lüthy, H., White, R.A., Sherby, O.D. (1979) Grain boundary sliding and deformation mechanisms maps. Mat. Svi. Eng. Vol. 39 (2), 211-216 https://doi.org/10.1016/0025-5416(79)90060-0

[7], Fazan, B., Sherby, O.D., Dorn, J.E. (1954) Some observations on grain boundary shearing during creep. JOM, vol. 6, 919-922.

[8], Bieler, T.R.; Eseinlohr, P.; Phukan, H.J.; Crimp, M.A. (2014) Grain boundaries and interfaces in slip transfer. Current Opinion in Solid State and Materials Sci., Vol 18 (4), 212-226. https://doi.org/10.1016/j.cossms.2014.05.003

[9], Bieler, T.R.; R.Alizadeh; M.Peña-Ortega; J.Llorca (2019) An analysis of (the lack of) slip transfer between near-cube oriented grains in pure Al, International Journal of Plasticity, 118, Pages 269-290. https://doi.org/10.1016/j.ijplas.2019.02.014

[10], Linne, M.A., Venkataraman, A., Sangid, M.D. et al. (2019) Grain Boundary Sliding and Slip Transmission in High Purity Aluminum. Exp Mech 59, 643-658. https://doi.org/10.1007/s11340-019-00517-z

[11], Alizadeh, R.; M.Peña-Ortega; T.R.Bieler; J.LLorca (2020) A criterion for slip transfer at grain boundaries in Al. Scripta Materialia, 178, 408-412, https://doi.org/10.1016/j.scriptamat.2019.12.010

[12], M. Bourcier, M. Bornert, A. Dimanov, E. Heripre, J. L. Raphanel, (2013) Multiscale experimental investigation of crystal plasticity and grain boundary sliding in synthetic halite using digital image correlation, Journal of Geophysical Research-Solid Earth 118 (2) 511-526. doi:\{10.1002/jgrb.50065\}.

[13], Gaye, A., Bourcier M., Bornert, A. Héripré, E., Dimanov, A., Raphanel, J. Sab, K. (2014), Micromechanics of Halite investigated by 2D and 3D Multiscale Full-Field Measurements. Am. Rock Mech Assoc.: ARMA, 48th US Rock Mechanics/Geomechanics Symposium, Mineapolis (USA).

[14], Combe, N.; Mompiou, F. and Legros, M. (2019) Heterogeneous disconnection nucleation mechanisms during grain boundary migration. Phys. Rev. Materials, 3 (6) 060601, https://doi.org/10.1103/PhysRevMaterials.3.060601

[15], Dautriat J; Bornert M; Gland N; Dimanov A, Raphanel J. (2011), Localized deformation induced by heterogeneities in porous carbonate analysed by multi-scale digital image correlation, Tectonophysics, Volume: 503(1-2) Special Issue: SI, 100-116 DOI: 10.1016/j.tecto.2010.09.025 
[16], Héripré, E.; Dexet, M.; Crépin, J.; Gélébart, L.; Roose, A.; Bornert, M. and Caldemaison, D. (2009) Coupling between experimental measurements and polycrystal finite element calculations for micromechanical study of metallic materials. International Journal of Plasticity 23(9):1512-1539, DOI: 10.1016/j.ijplas.2007.01.009

[17], Goyal A., Doquet V. and Pouya A. (2020) Grain Boundary Sliding and Strain Rate Sensitivity of Coarse and Fine/Ultrafine Grained 5083 Aluminum Alloys Metallurgical and Materials Transactions A volume 51, p1109-1122, , DOI:10.1007/s11661-019-05583-5

[18], M. A. Sutton, N. Li, D. C. Joy, A. P. Reynolds and X. Li (2007), Scanning electron microscopy for quantitative small and large deformation measurements part i: Sem imaging at magnifications from 200 to 10,000 , Experimental Mechanics 47 (6) 775-787. doi:10.1007/s11340-007-9042-z.

[19], Doumalin, P., M. Bornert, D. Caldemaison (1999), Microextensometry by image correlation applied to micromechanical studies using the scanning electron microscopy, in: Proc. Int. Conf. on Advanced Techno-logy in Experimental Mechanics, Vol. I, Atem 99, The Japan Societyof Mechanical Engineering, 1999, pp. 81-86.

[20], Doumalin, P. and M. Bornert (2000), Micromechanical applications of digital image correlation techniques, in: P. Jacquot, J.M. Fournier (Eds.), Proc. Interferometry in Speckle Light; Theory and Applications, EPFL, Lausanne, Springer-Verlag, Berlin, pp. 67-74.

[21], Lens, A., C. Maurice, J. Driver (2005), Grain boundary mobilities during recrystallization of Al-Mn alloys as measured by in situ annealing experiments, Material Science and Engineering A-Structural Materials Properties Microstructure and Processing 403 (1-2), 144-153. doi:\{10.1016/j.msea.2005.05. 010\}.

[22], R. Quey, D. Piot, J. H. Driver (2010), Microtexture tracking in hot-deformed polycrystalline aluminium: Experimental results, Acta Materialia 58 (5) 1629-1642. doi:\{10.1016/j.actamat.2009.11.007\}.

[23], Mecif, A. B. Bacroix, P. Franciosi, (1997), Temperature and orientation dependent plasticity features of Cu and Al single crystals under axial compression .1. Lattice rotation effects and true hardening stages, Acta Materialia 45 (1) 371-381. doi: $\{10.1016 / \mathrm{S} 1359-6454(96) 00094-8\}$.

[24], El Sabbagh, A. (2018) Charactérisation du mécanisme de glissement aux joints de grains dans l'aluminium à haute température par mesures de champ in situ MEB. Thèse de Doctorat en Mécanique des Matériaux de l'Ecole Polytechnique, Palaiseau.

[25], Bacroix, B., J.J., Jonas (1987) The Influence of Non-Octahedral Slip on Texture Development in FCC Metals. Texture, Stress, and Microstructure, Volume 8 |Article ID 957619 | https://doi.org/10.1155/TSM.8-9.267

[26], Couret, A., D. Caillard, (1988) Microscopic observations of glide in non close-packed planes in aluminium, and comparison with magnesium, Acta Metallurgica 36 (9) 2515 - 2524. doi:https://doi.org/10. 1016/00016160(88)90197-6.

[27], Caillard, D., J-L., martin (1989) Some aspects of cross-slip in metals and alloys. Journal de Physique, 1989, 50 (18), pp.2455-2473. 10.1051/jphys:0198900500180245500

[28], Albou, A., A. Borbely, C. Maurice, J. H. Driver, (2011) Orientation-dependent recovery in strongly deformed A10.1\% Mn crystals, Philosophical Magazine 91 (31) 3981-4000. doi:\{10.1080/14786435.2011. 600733$\}.$

[29], Caillard, D., Martin, J.-L. (2009) Glide of Dislocations in non-octahedral Planes of FCC Metals: A Review. International Journal of Materials Research, 100(10), DOI:10.3139/146.110190

[30], Picard, D., A. Dimanov, J. Raphanel (2018) Plastic behavior of halite single-crystals at different temperatures and strain rates: New insights from in-situ experiments and full field measures. Mat. Sci. Eng. A. Vol. 732, DOI:10.1016/j.msea.2018.07.009 
[31], Alquist C. N., R. A. Menezes, (1971) “Grain Boundary Sliding and the Observation of Superplasticity in Pure Metals," Mater. Sci. Eng. 7, 223-224

[32], Langdon, T. G. (1971) “Grain Boundary Sliding as a Deformation Mechanism During Creep,” Phil. Mag. 21, 689-700

[33], Ashby M. F., R. A. Verall, (1973) “Diffusion-Accommodated Flow and Superplasticity,” Acta Met. 21, 149163

[34], R. Raj and M. F. Ashby, “On Grain Boundary Sliding and Diffusional Creep,” Met. Trans. 2, 1113-1127 (1971).

[35], Rajabzadeh, A., F. Mompioua, S. Lartigue-Korinekc, N. Combe, M. Legros, D.A. Molodov (2014) The role of disconnections in deformation-coupled grainboundary migration, Acta Materialia, 77, 223-235

[36], Nicolas Combe, Frédéric Mompiou, Marc Legros. (2016) Disconnections kinks and competing modes in shear-coupled grain boundary migration. Physical Review B: Condensed Matter and Materials Physics (1998-2015), American Physical Society, 93 (2), pp.024109. 〈10.1103/PhysRevB.93.024109〉.

[37], Nicolas Combe, Frédéric Mompiou, M Legros. 2019. Heterogeneous disconnection nucleation mechanisms during grain boundary migration. Physical Review Materials, American Physical Society, 3 (6),10.1103/PhysRevMaterials.3.060601.

[38], M. Larranaga, F. Mompiou, M. Legros, and N. Combe. (2020) Role of sessile disconnection dipoles in shearcoupled grain boundary migration Phys. Rev. Materials 4, 123606

[39], Mussot, P., C. Rey and A. Zaou, 1985, Grain Boundary Sliding and Strain Compatibility, Res. Mechanica 14 (1985) 69-79

[40], Gaël Daveau, 2012. Interaction dislocations - joints de grains en déformation plastique monotone : étude expérimentale et modélisations numériques. Thèse Ecole Centrale, Paris 